Westra et al.

\title{
1 Fine-mapping identifies causal variants for 2 RA and T1D in DNASE1L3, SIRPG, MEG3, TNFAIP3 and CD28/CTLA4 Ioci
}

Harm-Jan Westra ${ }^{1,2}$, Marta Martinez Bonet ${ }^{3}$, Suna Onengut ${ }^{4,5}$, Annette Lee ${ }^{6}$, Yang Luo ${ }^{1,2}$, 6 Nick Teslovich $^{1,2}$, Jane Worthington ${ }^{7}$, Javier Martin ${ }^{8}$, Tom Huizinga ${ }^{9}$, Lars Klareskog ${ }^{10}$, 7 Solbritt Rantapaa-Dahlqvist ${ }^{10,11}$, Wei-Min Chen ${ }^{4,5}$, Aaron Quinlan ${ }^{4,12}$, John A. Todd ${ }^{13}$, 8 Steve Eyre ${ }^{7}$, Peter A. Nigrovic ${ }^{3,14}$, Peter K. Gregersen ${ }^{6}$, Stephen S Rich ${ }^{4,5}$, Soumya 9 Raychaudhuri ${ }^{1,2,3,14,15, *}$

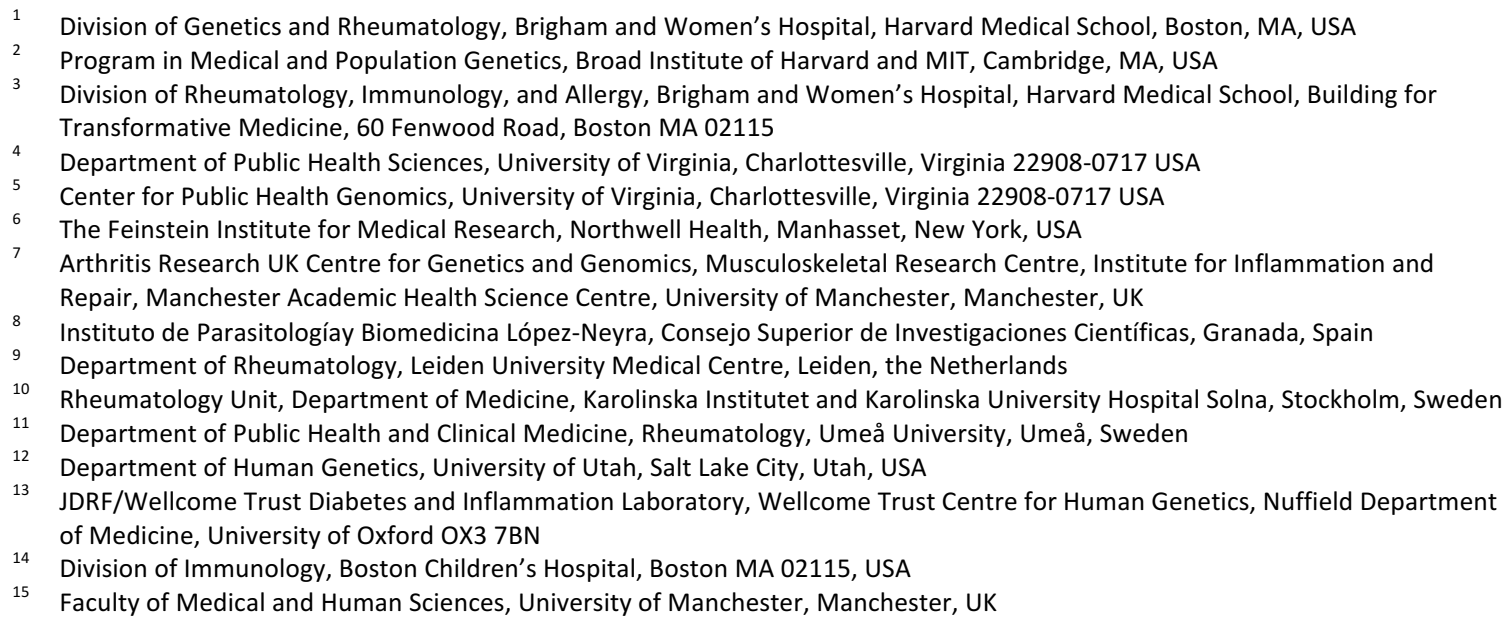

* Correspondence to:

31 Soumya Raychaudhuri

3277 Avenue Louis Pasteur, Harvard New Research Building, Suite 250D

33 Boston, MA 02446, USA.

34 soumya@broadinstitute.org; 617-525-4484 (tel); 617-525-4488 (fax) 
Westra et al.

35 We fine-mapped 76 rheumatoid arthritis (RA) and type 1 diabetes (T1D) loci

outside of the MHC. After sequencing 799 1 kb regulatory (H3K4me3)

regions within these loci in 568 individuals, we observed accurate

38 imputation for $89 \%$ of common variants. We fine-mapped ${ }^{1,2}$ these loci in RA

$(11,475 \text { cases, } 15,870 \text { controls })^{3}$, T1D $(9,334 \text { cases and } 11,111 \text { controls })^{4}$ and

combined datasets. We reduced the number of potential causal variants to

$41 \leq 5$ in 8 RA and 11 T1D loci. We identified causal missense variants in five

42 Ioci (DNASE1L3, SIRPG, PTPN22, SH2B3 and TYK2) and likely causal noncoding variants in six loci (MEG3, TNFAIP3, CD28/CTLA4, ANKRD55, IL2RA,

44 REL/PUS10). Functional analysis confirmed allele specific binding and

45 differential enhancer activity for three variants: the CD28/CTLA4

rs117701653 SNP, the TNFAIP3 rs35926684 indel, and the MEG3 rs34552516

indel. This study demonstrates the potential for dense genotyping and imputation to pinpoint missense and non-coding causal alleles.

$50 \mathrm{RA}$ is an autoimmune disease in which chronic inflammation leads to joint

51 destruction, which is associated with autoantibodies to citrullinated proteins in

52 the majority of cases ${ }^{5}$. T1D arises through autoimmune destruction of pancreatic

53 beta-cells, leading to complete loss of insulin production. Autoantibodies in T1D

54 include those reactive to proinsulin ${ }^{6}$ and glutamic acid decarboxylase ${ }^{7}$. Genome

55 wide association studies (GWAS) have identified over $101 \mathrm{RA} \mathrm{loci}^{3,8}$ and 53 T1D

56 loci $^{4}$. In order to define causal variants, fine-mapping has now been successfully 
Westra et al.

57 applied to complex disease loci including inflammatory bowel disease ${ }^{9}$, type 2

58 diabetes $^{1,10}$, coronary artery disease ${ }^{1}$, Graves disease $^{1}$, and multiple sclerosis ${ }^{11}$.

59 Since causal variants for both RA and T1D diseases overlap functional elements

60 in CD4+ T cells ${ }^{12}$, we fine-mapped autosomal non-MHC loci for both diseases

61 together.

63 We used ImmunoChip data for RA (11,475 cases, 15,870 controls) ${ }^{3}$, and T1D

$64(9,334 \text { cases and 11,111 controls; Supplementary Table } 1)^{4}$. This platform

65 contains dense coverage of single nucleotide polymorphisms (SNP) in selected

66 autoimmune disease loci, enabling accurate imputation. Among these loci, 46

67 and 49 non-MHC autosomal loci have known significant associations for RA and

68 T1D, respectively. Since RA and T1D share 19 loci, we examined 76 unique

69 ImmunoChip loci in total (Supplementary Table 2).

71 We used three high-quality reference panels and selected the imputation

72 strategy that maximizes coverage and accuracy for common variants (minor

73 allele frequency; MAF>1\%): 1) the Haplotype Reference Consortium (HRC, v1.1)

74 reference panel (consisting of 64,976 haplotypes from 20 independent

75 sequencing studies ${ }^{13}$ ), 2) the 1000 genomes (1KG, 3v5) European subpopulation

76 (EUR) and 3) the 1KG cosmopolitan panel (COSMO) ${ }^{14}$. To evaluate accuracy of

77 each strategy, we sequenced 568 individuals genotyped on ImmunoChip,

78 targeting 7991,000 bp regions centered around H3K4me3 peaks in 
Westra et al.

79 ImmunoChip regions (Online Methods). From this data, we called 1,862 variants

80 (MAF>1\%; Supplementary Figure 1; Supplementary Table 3), which we

81 compared to imputed genotypes. EUR and COSMO provided higher accuracy,

82 compared to the HRC (89\% vs $84 \%$ of variants with $r_{g}^{2}>0.5$; Figure 1A\&B,

83 Supplementary Tables 4-5). Imputation with COSMO obtained $1.8 \%$ higher

84 coverage for variants with high quality (INFO>0.3) variants than with EUR

85 (Supplementary Table 6). The difference between COSMO and HRC was

86 partially due to the inclusion of insertion/deletion variants (indels) in COSMO

87 (Supplementary Figure 2 and 3). INFO-scores were consistent with imputation

88 accuracy (Supplementary Figure 4). We therefore opted to use COSMO to

89 impute genotypes.

91 Notably, even this best performing strategy had incomplete variant coverage:

$924 \%$ of common variants in the targeted sequencing experiment were missed

93 altogether, of which $75 \%$ were indels and multi-allelic variants (Supplementary

94 Figure 5).

We focused our analysis on a subset of the loci with a tractable number of

97 putative causal variants within our data set. First, we calculated association

98 statistics for 64,430 and 66,115 imputed and genotyped variants for RA and T1D

99 (MAF>1\%, INFO>0.3; Hardy-Weinberg $\left.p>10^{-5}\right)$ in the 76 loci. We observed

100 association in 20 and 36 loci, for RA and T1D $\left(p<7.5 \times 10^{-7}=0.05 / 66,115\right.$ tests; 
Westra et al.

101 Supplementary Table 7 and 8$)$. For $50 \%(=10 / 20)$ of RA and $72 \%(=26 / 36)$ of

102 T1D loci, the most significant variant was in linkage disequilibrium (LD; $\left.r^{2}>0.8\right)$

103 with the most significant previously published variant (Supplementary Table 7

104 and 8). RA and T1D variant effect sizes were positively correlated in $64 \%$ of the

105 tested loci (Online methods, Supplementary Table 9, Supplementary Figure

106 6) suggesting shared signals. We therefore analyzed a combined dataset with

10720,787 (RA or T1D) cases and 18,616 unique controls (Online methods). We

108 restricted our analysis to 28 loci with sufficient statistical signal to warrant fine-

109 mapping in the combined dataset $\left(p<7.5 \times 10^{-7}\right)$. In the combined dataset, the

110 strongest associated variant was in strong LD with the strongest associated

111 variant in either RA or T1D in $69 \%$ of significant loci $\left(r^{2}>0.8\right.$; Supplementary

112 Table 10 and 11). To prioritize loci with causal variation that we might be able to

113 pinpoint, we created $90 \%$ credible sets using an approximate Bayesian

114 approach $^{1,2}$ and limited subsequent analysis to the 10 (RA), 15 (T1D) and 11

115 (combined) loci having $\leq 10$ variants in the $90 \%$ credible sets (Figure 2A\&B;

116 Supplementary Table 12). Within the significant loci, we observed a striking

117 18.3-fold posterior probability enrichment for missense variants.

119 We identified those alleles as likely causal if they had strong statistical genetic

120 evidence and evidence of altered function (Table 1). To define strong candidate

121 alleles, we defined three overlapping categories of promising loci: loci with 1) a

122 single variant with a very high posterior probability $(>0.8, D N A S E 1 L 3, P T P N 22$, 
Westra et al.

123 TYK2, CTLA4/CD28, REL/PUS10, IL2RA), 2) a single missense variant with a

124 modest posterior probability (>0.2, DNASE1L3, PTPN22, SH2B3, TYK2, SIRPG),

125 or 3$)$ a single non-coding indel with a modest posterior probability $(>0.2$,

126 TNFAIP3, MEG3, ANKRD55; Figure 2C; Supplementary Table 12). We applied

127 more modest thresholds to missense variants and indels, since they are a priori

128 more likely to be functional. We considered high probability non-coding variants

129 causal only if they met stringent additional criteria criteria suggesting function: 1)

130 they occurred in a region with evidence of enhancer activity and 2) they

131 demonstrated clear allele specific binding and enhancer function in vitro in both

132 EMSA and luciferase assays.

134 We identified missense variants at DNASE1L3 and SIRPG. We also identified

135 causal missense variants at PTPN22, SH2B3, and TYK2, which are well

136 described in the literature ${ }^{4,15-17}$ (Supplementary Note and Supplementary

137 Figures 7-9). Their identification suggests the sensitivity of our approach is high.

139 The 3p14 DNASE1L3 locus, strongly associated with RA, but not T1D $(p>0.02$,

140 Supplementary Figure 10), had a missense variant with high posterior

141 probability. The previously reported ${ }^{3}$ lead SNP rs35677470 was included as one

142 of the 5 variants within the $90 \%$ credible set of causal variants $\left(p=1.7 \times 10^{-8}\right.$;

143 posterior=0.81; Supplementary Table 12), and encodes a R206C change in the 
Westra et al.

144 DNASE1L3 protein product. After conditioning on R206C, we observed no

145 evidence of independent risk variants ( $p>5 \times 10^{-4}$; Supplementary Table 13).

146 R206C has been implicated with systemic sclerosis ${ }^{18}$ and other loss of function

147 mutations in DNASE1L3 have been reported in familial forms of systemic lupus

148 erythematosus ${ }^{19}$. R206C is a loss of function allele that abolishes the protein

149 product's nuclease activity ${ }^{20}$.

151 Within the SIRPG locus, we identified a missense variant with high posterior

152 (rs6043409; $p=3.94 \times 10^{-10}$; posterior=0.25), causing a V263A substitution in the

153 SIRPG gene product (Supplementary Figure 11; Supplementary Table 12).

154 Conditional analysis using rs6043409 obviated the association signal in the

155 locus $\left(p>2 \times 10^{-3}\right)$. Since the consequence of V263A substitution on SIRPG

156 function has yet to be described, we nominate it as a causal variant with

157 caution.

159 Next, we focus on non-coding likely causal variants. We identified non-coding 160 allele specific function in CTLA4/CD28, TNFAIP3, and MEG3 using EMSA and

161 luciferase assays in regions with evidence of CD4+ T cell enhancer function

162 (Table 1). Loci having candidate variants with high posterior probabilities, but

163 without evidence of allelic function, are presented in the Supplementary Note 
Westra et al.

166 The CD28/CTLA4 locus has previously been shown to have shared association

167 signals for $\mathrm{RA}$ and $\mathrm{T}^{\mathrm{N}} \mathrm{D}^{21}$ and variant effect sizes between diseases are highly

168 correlated in our analysis (Spearman's rank r=0.9; Supplementary Table 9). In

169 the combined analysis, we observed a single credible variant (rs3087243;

$170 \mathrm{p}=1.4 \times 10^{-16}$; posterior $\left.=0.91\right)$ near $C T L A 4$. That same variant has the largest

171 posterior probability in T1D $\left(p=1.7 \times 10^{-15}\right.$; posterior=0.46; Figure 3A;

172 Supplementary Figure 15A; Supplementary Table 12), but not in RA

$173\left(p=1.6 \times 10^{-8} ;\right.$ posterior $\left.=0.01\right)$. In contrast, in RA the rs 117701653 variant carries

174 high posterior probability $\left(p=1.3 \times 10^{-10}\right.$; posterior $\left.=0.82\right)$; it is located closer to

175 CD28 and is not linked to $r$ 3087243 $\left(r^{2}=0.03\right)$. Conditioning on rs3087243, we

176 observed an independent effect at rs117701653 in RA $\left(p=1.8 \times 10^{-8}\right)$, (Figure 3A;

177 Supplementary Table 13). To confirm the two independent effects, we tested

178 all pairs of SNPs exhaustively and observed that the rs3087243+rs117701653

179 pair demonstrates the most significant association of all SNP pairs in RA (Figure

180 3B, Supplementary Figure 15B). Haplotype analysis confirmed the

181 independent protective effects of the rs3087243 A allele and of the rs117701653

182 C allele in both RA and T1D (Figure 3C), suggesting that rs117701653 may

183 contribute to risk similarly in T1D ( $p=0.03$ in conditional haplotype analysis). 
Westra et al.

185 We observed that both rs117701653 and rs3087243 may have regulatory

186 function since they overlap H3K4me3 peaks in immune cell types, and disrupt

187 protein binding motifs (Supplementary Table 14 and 15). Since regulatory

188 regions can be context specific, we stimulated CD4+ T cells using CD3/CD28

189 beads, and measured chromatin accessibility using ATAC-seq before and after

190 stimulation. We observed ATAC-seq peak overlap for rs117701653 only after

191 stimulation (Supplementary Table 16), suggesting that rs117701653 may

192 function specifically in stimulated cells. We note that while we did not observe

193 linkage to eQTL in whole blood or T cells (Supplementary Table 17), rs3087243

194 did show a significant eQTL on CTLA4 in testis ${ }^{22}$.

We demonstrated allele specific binding for rs117701653 but not rs3087243

197 using EMSA with Jurkat T cells (Figure 3D). The rs117701653 C allele showed

198 higher specific binding affinity compared to the A allele (Supplementary Figure

15C). We also observed higher luciferase expression induced by the $C$ allele

200 compared to the A allele ( $p=0.0017$; Figure 3E), suggesting allele specific

201 enhancer activity. The binding is lineage specific: it was absent with THP-1

202 monocytic cells (Supplementary Figure 15C). As a relevant negative control,

203 we also tested the second variant in the RA credible set (rs55686954;

204 posterior=0.14), which showed no evidence of allele specific enhancer function

205 (Supplementary Figure 15C\&D). Published promoter capture Hi-C assays ${ }^{23}$

206 show local genomic contacts between the region harboring the rs117701653 
Westra et al.

207 SNP, the CTLA4 promoter, and a region downstream of $R A P H 1$

208 (Supplementary Figure 16), indicating this allele might be regulating CTLA4 or

209 RAPH1 despite proximity to CD28.

211 The TNFAIP3 locus is associated with multiple autoimmune diseases ${ }^{24-30}$,

212 including RA, but not T1D $\left(p>2.3 \times 10^{-4}\right)$. We observed that the indel rs35926684

213 carries the highest posterior probability $\left(p=6.8 \times 10^{-12}\right.$; posterior=0.24; Figure 4A;

214 Supplementary Table 12; Supplementary Figure 17A) of 7 variants in the RA

215 credible set. Conditional analysis revealed an independent association at

216 rs58721818 $\left(p=3.6 \times 10^{-5} ;\right.$ LD $R^{2}=0.05$ with rs35926684; Figure 4A;

217 Supplementary Table 13). A previous study ${ }^{3}$ identified rs6920220 (linked to

218 rs35926684; $r^{2}=0.88$ ) as the primary signal and secondary signals from

219 rs5029937 (linked to rs58721818; $r^{2}=0.84$ ) and rs13207033. Exhaustive pairwise

220 analysis demonstrated comparable association for rs35926684+rs58721818 pair

$221(-\log 10(p)=13.94)$ and the most strongly associated rs6920220+rs58721818 pair

$222 \quad(-\log 10(p)=14.14 ;$ Figure 4B; Supplementary Figure 17B). Haplotypes having

223 the rs35926684 G allele increased risk for RA, even in absence of the highly

224 linked rs6920220 A risk allele (i.e. GGGC vs GAGC; Figure 4C), although this

225 effect was only suggestive in conditional haplotype analysis $(p=0.14)$. 
Westra et al.

227 The rs35926684 indel alters more binding motifs, and overlaps more enhancer

228 marks in immune related cell types, compared to rs6920220 (Supplementary

229 Table 14 and 15). Neither rs35926684 nor rs6920220 overlapped open

230 chromatin region in our ATAC-seq time course (Supplementary Table 16), nor

231 were linked with eQTLs in whole blood or T-cells (Supplementary Table 17).

233 EMSA with Jurkat cells demonstrated specific binding for rs35926684 (Figure

234 4D). Dose titration of the probe demonstrated specific binding for both $\mathrm{G}$ and

235 GA allele, but stronger GA binding (Supplementary Figure 17C). Luciferase

236 assays also demonstrated increased enhancer activity with the GA-allele

237 compared to both the empty vector $\left(p=7 \times 10^{-4}\right)$ and the G allele $(p=0.053$, Figure

238 4E). We did not observe specific binding with THP-1 cells, indicating cell type

239 specificity (Supplementary Figure 17C). As a relevant negative control, we

240 observed no allele specific binding for rs6920220 (Supplementary Figure 17C).

241 Interestingly, in previously published promoter capture $\mathrm{Hi}-\mathrm{C}$ data, the

242 rs35926684 region contacts the TNFAIP3 promoter ${ }^{31}$ as well as the IL22RA and

243 IFNGR1 promoters (Supplementary Figure 16) ${ }^{23}$, suggesting genes with

244 immune function may be influenced by this RA risk allele.

246 MEG3 is a non-coding RNA tumor suppressor gene whose transcript binds to

$247 \mathrm{p} 53^{32}$. In T1D, this locus has previously been described as an imprinted region, 
Westra et al.

248 with greater risk carried by paternally inherited alleles ${ }^{33}$. We observed two

249 variants in the credible set for T1D in this locus: the rs34552516 indel

$250\left(p=1.1 \times 10^{-9} ;\right.$ posterior $\left.=0.37\right)$ and the $r 556994090$ intronic variant $\left(p=7.3 \times 10^{-10}\right.$

251 posterior=0.54, Figure 5A; Supplementary Figure 18A; Supplementary Table

252 12). The locus shows no association with $R A(p>0.04)$. Conditioning on

253 rs34552516, we observed no evidence of additional independent effects

254 ( $p>0.04$; Supplementary Table 13). Both variants overlap DNAse-I sensitive,

$255 \mathrm{H} 3 \mathrm{~K} 4 \mathrm{me} 1$, and H3K4me3 regions in multiple immune cell types (Supplementary

256 Table 14), but do not overlap open chromatin regions in our ATAC-seq

257 experiment (Supplementary Table 16).

259 EMSA with Jurkat cells showed protein binding specific to the TC allele of rs34552516 (Figure 5B), and the rs34552516 TC allele showed a significant

261 increase in luciferase activity compared to empty vector $(p=0.01)$ and the T allele

262 ( $p<0.05$; Figure 5C). We observed no specific binding with THP-1 cells

263 (Supplementary Figure 18B), indicating lineage specificity (Figure 5B). As a

264 relevant negative control, we did not observe allele specific binding for

265 rs56994090. The region harboring rs34552516 in promoter-capture Hi-C data ${ }^{23}$

266 showed contacts, including the promoter of DIO3 and RP11-1029J19

267 (Supplementary Figure 16), indicating that this risk allele may affect interaction

268 with multiple downstream genes. 
Westra et al.

270 In this study, we identified three non-coding causal alleles with high posterior

271 probability based on association data, and evidence of allele specific binding or

272 enhancer function (Table 1). We observed in targeted sequencing that a

273 proportion of causal variants might be missed by any imputation strategy,

274 particularly indels or multiallelic variants. We therefore recognize that attempting

275 to fine-map other loci may be more successful once more complete reference

276 panels based on whole genome sequencing data become available, such as

277 through the TopMed initiative (https://www.nhlbiwgs.org/).

279 Notably, the non-coding causal variants that we identified did not overlap with

280 eQTL in either whole blood or T cells (Supplementary Table 17). Therefore, to

281 elucidate the mechanisms underlying these variants, studies will be required to

282 identify the precise protein complexes that bind these enhancers, and the

283 downstream functions of those complexes.

285 We also identified other non-coding variants with high posterior probabilities

286 that could feasibly be pursued for validation, but did not demonstrate clear

287 evidence of allele-specific function in our assays. Other more sensitive assays,

288 or application of assays in other non-CD4+ T cell-types might ultimately be able

289 to confirm the function of these alleles too. 
Westra et al.

293

\section{Data Availability}

Summary statistics for all variants will be made available upon acceptance. Genotype data is previously published ${ }^{3,4}$ and is available from RACl and T1DGCC upon request. ATAC seq data will be deposited upon acceptance of this manuscript to GEO.

Bios eQTL browser: http://genenetwork.nl/biosqtlbrowser/, Roadmap epigenomics datasets: http://www.roadmapepigenomics.org/, ChromHMM enhancers and promotors: http://egg2.wustl.edu/roadmap/web_portal/, 1000 genomes reference panel: http://bochet.gcc.biostat.washington.edu/beagle/1000_Genomes_phase3_v5a/, Haplotype Reference Consortium panel: http://www.haplotype-referenceconsortium.org/

\section{Code Availability}

Associated computer code for this manuscript can be found at the following GitHub repositories:

https://github.com/immunogenomics/harmjan/tree/master/FinemappingPaper and https://github.com/immunogenomics/harmjan/tree/master/FinemappingTools

\section{Author Contributions}

Analysis: H-J.W., Y.L., S.R.; Functional Assays: M.M.B., P.A.N.

Study Design: H-J.W., P.A.N., S.R.; Data Acquisition: S.O., A.L., N.T., J.W., J.M., T.H., L.K., S.R-D., W-M. C., A.Q., J.A.T., P.K.G., S.S.R., S.R.; Writing and editing manuscript: H-J.W., M.M.B., Y.L., J.A.T., P.A.N., P.K.G, S.S.R., S.R 
Westra et al.

\section{Acknowledgements}

318 This work is supported in part by funding from the National Institutes of Health

319 (U01GM092691, UH2AR067677, 1U01HG009088, and 1R01AR063759 (SR)),

320 and the Doris Duke Charitable Foundation Grant \#2013097. This work is part of

321 the research program Rubicon ALW with project number \#825.14.019 (HJW),

322 which is (partly) financed by the Netherlands Organization for Scientific

323 Research (NWO). Further support was provided by the Wellcome Trust

324 [107212/Z/15/Z] and JDRF [5-SRA-2015-130-A-N] to the Diabetes and

325 Inflammation Laboratory and by the Wellcome Trust [203141/Z/16/Z] to the

326 Wellcome Trust Centre for Human Genetics. PKG was supported in part by the

327 Feinstein Institute and a generous gift from Eileen Ludwig Greenland (PKG). PAN

328 is supported by a Rheumatology Research Foundation Disease Targeted

329 Research Grant, NIH P30 AR070253, and the Fundación Bechara.

\section{Competing financial interests}

332 None declared 
Westra et al.

\section{Online Methods}

\section{Patient collections}

335 We used genotyping data from samples that were collected on the ImmunoChip

336 platform, which were obtained with informed consent and described in previous

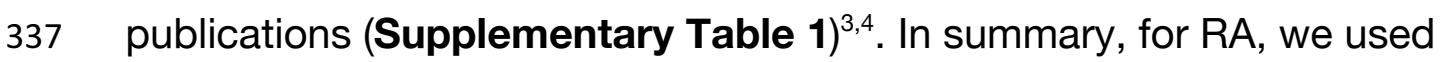

338 ImmunoChip data for 11,475 cases and 15,870 controls, collected by six

339 different cohorts (UK, Swedish EIRA, United States, Dutch, Swedish UMEA, and

340 Spanish) $)^{3}$. For T1D, we used ImmunoChip data for 12,241 cases and 14,636

341 controls divided over two different cohorts, that have been described earlier:

342 the T1DGC family collection (T1D EUR) and the UK GRID, British 1958 Birth

343 cohort and UK Blood Service collection (T1D UK). In order to include trios from

344 the T1D EUR cohort in a case-control analysis, we generated pseudocontrol

345 pairs for each affected individual using the untransmitted alleles from the

346 parents of that individual. As a consequence, the final number of individuals for

347 T1D was 9,334 cases, and 11,111 controls (including 1,661 pseudocontrols).

348 Quality control on the genotypes was performed as described in the previously

349 published studies. Additionally, we merged the genotype data for the different

350 cohorts within T1D and RA using PLINK ${ }^{34}$, and converted genomic coordinates

351 using the UCSC liftOver tool and the hg18ToHg19 chain file. Variants unable to

352 liftOver were removed. We then replaced the variant identifiers using NCBI

$353 \mathrm{dbSNP}$ build 138. Finally, we removed variants with a MAF $<0.5 \%$. 
Westra et al.

\section{Imputation}

356 In order to assess which imputation strategy was best suited for fine-mapping,

357 we tested three reference panels: 1) The European subpopulation of 1000

358 genomes $(\mathrm{N}=503), 2)$ the cosmopolitan panel of 1000 genomes $(\mathrm{N}=2,504)$, and

359 3) the HRC v1.1 reference panel $(\mathrm{N}=32,611)$. Our approach used three steps

360 (matching, imputation, and merging). First, we matched variants to each

361 reference panel: we removed variants that were not present in the reference

362 panel and aligned the strands of the remaining ImmunoChip genotypes. We

363 excluded variants when alleles could not be matched, or in the case of $\mathrm{C} / \mathrm{G}$ and

364 A/T variants, when the minor allele was unequal. If we observed an unequal

365 minor allele for such variants, and the reference panel and ImmunoChip MAF

366 was $>45 \%$, we chose to flip the allele in the ImmunoChip data. For multi-allelic

367 variants, we ensured that the allele encoding was identical relative to the

368 reference panel variant. As a consequence of these steps, the input for each

369 reference panel was slightly different (Supplementary Table 4). Second, we

370 imputed genotypes into RA and T1D separately. We phased and imputed the

3711000 genomes reference panels using Beagle v4.1 (version 22Apr16.1cf) ${ }^{35}$. In

372 order to accommodate computational constraints of Beagle, we split the RA and

373 T1D datasets into 30 batches, randomizing cases and controls between

374 batches, while maintaining trio structure in the T1D dataset. Since the HRC v1.1

375 reference panel genotype data is not publicly available, we evaluated different

376 imputation servers and settings for the T1D dataset, in order to determine their 
Westra et al.

377 effects on imputation output. On the Sanger Institute imputation server (date of 378 access: May 11, 2016), we used prephasing with either EAGLE ${ }^{36}$ or SHAPEIT $^{37}$, 379 followed by imputation with $\mathrm{PBWT}^{38}$. On the Michigan University server (date of 380 access: July 5, 2016), we used prephasing with EAGLE ${ }^{36}$, followed by MiniMac ${ }^{39}$

381 imputation. Due to the constraints of the Michigan University imputation server 382 website, we split the dataset into three batches, randomizing cases and controls 383 while maintaining trios. For RA, we performed HRC imputation on the Sanger 384 imputation server using EAGLE prephasing followed by PBWT imputation. Third, 385 we merged the imputed dosages and probabilities from each batch (if any) for 386 each imputation reference panel, and replaced the variant identifiers in the

387 imputed output using NCBI dbSNP build 138. Before calculating association 388 statistics, we replaced genotypes for variants genotyped on ImmunoChip with 389 the original genotypes. Finally, we recalculated the imputation quality scores for each imputed variant in each dataset: for biallelic variants, we used the INFO 391 score and Beagle v4.1 allelic- $\mathrm{R}^{2}$ for multi allelic variants.

\section{Targeted sequencing}

394 In order to test the accuracy of imputation, we sequenced targeted regions in 395864 individuals (160 T1D trios and 384 unrelated RA cases, of which 480 and 396149 were on ImmunoChip, respectively). We used the Illumina MiSeq platform to generate $100 \mathrm{bp}$ paired-end reads. We sequenced 900 regions of $1,000 \mathrm{bp}$

398 around H3K4me3 peaks centers overlapping loci associated with either disease, 
Westra et al.

399 since these loci are most likely to harbor causal variants ${ }^{12}$. We used BWA-mem ${ }^{40}$

400 (v0.7.12) to align reads to the hg19 reference genome. We tagged and removed

401 duplicate reads using Picard MarkDuplicates. We then removed 101 regions

402 where $>50 \%$ of the samples had $<20 x$ coverage at $>80 \%$ of sequenced bases,

403 and removed 86 samples having $<20 x$ coverage at $90 \%$ of sequenced bases.

404 We called genotypes using GATK version 3.4, following the recommended

405 guidelines for using HaplotypeCaller ${ }^{41}$ in a joint genotype calling approach. We

406 then set genotypes with $<10 x$ coverage and QUAL $<30$ to missing, and excluded

407 variants with $>5 \%$ missingness. We corrected for possible sample swaps and

408 mismatched samples by correlating the called genotypes with ImmunoChip

409 genotypes and removing samples that did not match any ImmunoChip sample

410 ( $r<0.95)$, resulting in 568 final samples (including 439 for T1D, and 129 for RA).

411 Finally, we selected variants with MAF>1\%, resulting in 1,862 variants within the

$41276 \mathrm{RA}$ and T1D associated regions.

\section{$414 \quad$ Merging imputed datasets}

415 Prior to the association analysis, we merged the data for the RA and T1D

416 dataset, imputed with the COSMO reference panel. Since these cohorts share

417 controls, not necessarily with identical identifiers, we first identified individuals

418 with high shared genetic background. For this purpose, we first generated a list

419 of LD pruned variants from the ImmunoChip genotypes using PLINK ${ }^{34}$ (using --

420 indep-pairwise 1000100 0.2). We then used this list to determine the genetic 
Westra et al.

421 similarity (unified additive relationship; UAR) ${ }^{42}$ between each pair of samples

422 across both datasets. We considered sample pairs with an UAR $>0.2$ genetically

423 related and randomly selected one sample of the pair to be included from either

424 the RA or the T1D dataset. We considered the remaining sample pairs unrelated.

425 We finally merged genotypes and imputation probabilities from the selected

426 samples, and recalculated the imputation INFO scores for the merged

427 genotypes as described earlier.

429 Association analysis framework

\section{$430 \quad$ Fine-mapping and statistical analysis}

431 Due to the sample size of the datasets in our study, we limited our association

432 analysis to variants having an overall MAF>1\%, a Hardy-Weinberg P-value

$433(\mathrm{HWE}-\mathrm{P})>10^{-5}$ in controls, and an overall INFO score $>0.3$. HWE-P was calculated

434 using an exact test for biallelic variants, while for multi-allelic variants, Pearson's

435 chi-squared test was applied. We then split multi-allelic variants into multiple

436 variants, creating a single variant for each alternate allele. To test each variant

437 for association with disease, we used logistic regression, assuming a log-linear

438 relation between the number of alternative alleles and case-control status. We

439 then created a null model containing covariates in order to account of

440 population differences. In the RA dataset, the null model included the first 10

441 principal components calculated over the genotype covariance matrix as

442 described earlier ${ }^{3}$, and included an additional 5 covariates indicating the 
Westra et al.

443 originating cohort. For T1D, we included 12 regional indicator variables in the

444 null model as described earlier ${ }^{4}$, and an additional variable indicating the

445 originating cohort. For each variant, we then fit an alternate model containing

446 the genotypes. For the joint analysis, the null model included all covariates for

447 the T1D and RA datasets and an additional covariate indicating whether the

448 sample originated either from the RA or the T1D dataset. In order to account for

449 imputation uncertainty, we recoded the imputation probabilities to a dosage

450 value ranging between 0 and 2 (i.e. $P(A B)+2 x P(B B)$ ). Finally, we calculated the

$451 \mathrm{p}$-value for the association as the difference in deviance between the alternative

452 and null models, which follows a chi-squared distribution with 1 degree of

453 freedom. To determine the significance of the association we calculated a

454 study-wide Bonferroni threshold using the maximum number of tests across

455 datasets $\left(p<7.5 \times 10^{-7}=0.05 / 66,115\right)$.

\section{Definition of credible sets}

458 To define the most likely causal variant for each locus, we calculated posterior

$459 \mathrm{p}$-values using the approximate Bayesian factor $(\mathrm{ABF})^{1,2}$ under the assumption

460 of a single causal variant per locus. Shortly, this framework assumes that the

461 association effect sizes follow a $\mathrm{N}(0, \mathrm{~V})$ distribution under $\mathrm{H}_{0}$, with $\mathrm{V}$ being the

462 standard error squared of the association. Under $\mathrm{H}_{1}$ the framework assumes a

463 distribution following $N(0, V+W)$, where $W$ is $(\ln (1.5) / 1.96)^{2}$, reflecting the prior of

464 observing an odds ratio of 1.5. The ABF for an observed effect size $B$ is then 
Westra et al.

465 calculated as the ratio of $\mathrm{P}\left(\mathrm{B} \mid \mathrm{H}_{0}\right) / \mathrm{P}\left(\mathrm{B} \mid \mathrm{H}_{1}\right)$, effectively measuring the probability of

466 observing the effect size under the $\mathrm{H}_{0}$ of no association over the $\mathrm{H}_{\mathrm{A}}$ of observing

467 an association. Using the sum of the ABF for all variants in the locus, we

468 calculate the posterior for variant i as:

$$
P_{i}=\frac{A B F_{i}}{\sum_{k=0}^{n} A B F_{k}}
$$

470 Following calculation of the posterior $p$-values, we created credible sets within

471 each locus by sorting associations descending on the basis of their posterior $\mathrm{p}$ -

472 values, and including associations such that the sum of their posteriors is $>0.9$.

\section{Detecting secondary associations}

475 In order to determine the presence of multiple independent effects, we

476 performed a conditional analysis using logistic regression: for each locus with a

477 significant association, we included the top-associated variant as a covariate in

478 the null model, and repeated the association analysis for that locus.

480 For each locus with a significant secondary association, we then tested whether

481 the observed pair of top-associated variants together provided the strongest

482 pairwise association signal given the variants in the locus by performing an

483 exhaustive pairwise analysis. Similarly to the normal logistic regression, the null

484 model included the covariates for each dataset, while the alternate model 
Westra et al.

485 included genotype dosages for both variants. The significance of the pairwise 486 association was then calculated using the difference in deviance between the

487 null and alternative models, following a chi-squared distribution with 2 degrees 488 of freedom.

490 Finally, for loci with two or more independent associations, we assessed 491 whether the risk alleles for the associated variants were located on the same 492 haplotypes. For the independently associated variants, we derived haplotypes 493 from the phased imputation output (e.g. 4 haplotypes for 2 independent 494 variants), and assigned two haplotypes to each individual. We then removed all 495 haplotypes with a frequency $<1 \%$, and removed all individuals that had any of 496 the removed haplotypes from the analysis. By using the haplotype with the 497 highest frequency as the reference haplotype, we assigned each individual to 498 have either 0,1 , or 2 copies of each alternative haplotype. We then used logistic 499 regression to test each haplotype for association, assuming a log-linear 500 relationship between the number of haplotype copies and disease status. To 501 correct for population differences, our null model included covariates as 502 described above. 
Westra et al.

505 eQTLs, H3K4me3 peaks, DNAse-I hypersensitive sites, enhancers and 506 motifs

507 In order to provide functional annotation for the identified variants, we assessed

508 overlap with eQTL, H3K4me3 peaks, DNAse-I hypersensitive sites, promoters

509 and enhancers. We used eQTL from a large RNA-seq based eQTL meta-

510 analysis using 2,116 whole blood samples ${ }^{43}$. Because many eQTL are cell type

511 specific, and RA and T1D loci are enriched for enhancers in CD4+ T cells ${ }^{12}$, we

512 also included a study assessing eQTL in CD4+ T cells using 461 individuals ${ }^{44}$.

513 For each variant in a credible set, we first determined whether the variant was

514 present in the eQTL summary statistics. We then selected the eQTL gene with

515 the lowest eQTL p-value. For variants that were not present, we selected the

516 eQTL snp with a linkage disequilibrium (LD) $r^{2}>0.8$, using the European

517 subpopulation in 1000 genomes as a reference panel. For eQTLs with equal LD,

518 we selected the eQTL gene with the lowest P-value.

520 We downloaded annotations in narrowPeak format for H3K4me3 peaks, DNAse-

521 I peaks, and ChromHMM ${ }^{45}$ genome segmentations from the Roadmap

522 epigenetics consortium ${ }^{46}$, consisting of 127 consolidated epigenomes from a

523 large number of different cell types. We then grouped immune related cell types

524 into an 'immune' group, and the remaining cell types in an 'other' group,

525 resulting in two groups for DNAse-I and H3K4me3 annotations. We additionally

526 used ChromHMM annotations created using 12 imputed epigenetic marks. 
Westra et al.

527 Additional to the 'immune' and 'other' groups, we further grouped ChromHMM

528 segments for enhancers (i.e. segments with an EnhA1, EnhA2, EnhW1, EnhW2,

529 and enHAc annotation) and promoters (i.e. segments with PromP, PromBiv,

530 PromU, PromD1 and PromD2 annotation), resulting in four annotation groups for

531 ChromHMM annotations. Within each group, we subsequently determined the

532 percentage of files in which we observed overlap between an annotation and

533 variants within the credible sets. Finally, we determined whether candidate

534 causal variants affected protein binding motifs or transcription factor binding

535 sites using HaploReg ${ }^{47}$.

537 The number of cell types in each group was different between annotations,

538 because not all annotations were present for all cell types. Numbers of files per

539 annotation group can be found in Supplementary Table 14.

$541 \quad$ ATAC-seq timeseries

542 ATAC-seq is a method to measure chromatin accessibility using a small number

543 of cells ${ }^{48}$. We here applied ATAC-seq to measure chromatin accessibility in a

544 timeseries after stimulation. We used $30 \mathrm{~mL}$ whole blood from a leukopak

545 acquired from a healthy anonymous donor in a 20mL PBS solution. We then

546 isolated PBMCs using Ficoll tubes and stored $500 \mu$ l aliquots of $100 \times 10^{6}$ cells in

547 liquid nitrogen. Cells were subsequently thawed, and stained with anti-biotin 
Westra et al.

548 microbeads for magnetic assisted cell sorting (MACS) to select CD4+ Tmem

549 cells. Cells were resuspended and transferred to a 24 wells plate in $3 \mathrm{ml}$ aliquots

550 of $6 \times 10^{6}$ cells. Cells were stimulated using Dynabeads (Human T-Activator

551 CD3/CD28 for T Cell Expansion and Activation; Life Technologies) in a 2 cells

552 per bead ratio. Samples of 100,000 cells were taken at $0,1,2,4,8,12$, 24, and

55348 hours after stimulation. Nucleosome isolation and ATAC-seq open chromatin

554 sequencing was performed as described earlier ${ }^{48}$. Sequenced reads were

555 mapped to the hg19 reference genome, using BWA-mem. Reads mapping to

556 the mitochondrial genome, reads mapping to multiple genomic locations, and

557 duplicate reads (labeled by Picard MarkDuplicates) were removed, and reads

558 were shifted +4 and -5 bp for the reverse and forward strands respectively.

559 Enrichment for open chromatin was determined by calling peaks using MACS

$560 \mathrm{v} 2^{49}$, using default settings.

562 Electrophoretic mobility shift assay

563 Cell lines

564 Lymphocytic and monocytic cell lines, Jurkat and THP-1 respectively, were

565 obtained from the ATCC (TIB-152 and TIB-202). Jurkat cells were grown in

566 complete RPMI (RPMI-1640, Gibco, with 10\% decomplemented-fetal bovine

567 serum, penicillin and streptomycin) and THP-1 cells in complete RPMI

568 supplemented with 2-mercaptoethanol to a final concentration of $0.05 \mathrm{mM}$. Both 
Westra et al.

569 cell lines were grown in a $37^{\circ} \mathrm{C}$ incubator with $5 \% \mathrm{CO} 2$.

\section{Electrophoretic mobility shift assay (EMSA)}

572 EMSA was performed using the LightShift Chemiluminiscent EMSA Kit (Thermo

573 Scientific). Single stranded oligonucleotides corresponding to a 30-32

574 nucleotides fragment of the human genome with the SNP of interest in the

575 middle were purchased from IDT (Supplementary Table 18). Single stranded

576 oligonucleotides were biotinylated using the Biotin 3'End DNA Labeling Kit

577 (Thermo Scientific) following manufacturer instructions. Double stranded

578 oligonucleotides were generated by mixing together equal amounts of biotin-

579 labeled (for probe) or unlabeled (for competitor) complementary oligonucleotides

580 and incubating them $5 \mathrm{~min}$ at $95^{\circ} \mathrm{C}$ and then 1 hour at room temperature.

582 Nuclear extract from Jurkat and THP-1 cells was obtained using the NE-PER ${ }^{\mathrm{TM}}$

583 Nuclear and Cytoplasmic Extraction Reagents (Thermo Scientific) following

584 manufacturer instructions. Protein extracts were dialyzed using a dialysis

585 membrane with MWCO of 12-14 KDa (Spectrum Spectra) against $1 \mathrm{~L}$ of dialysis

586 buffer (10 mM Tris pH 7.5, 50 mM KCl, 200 mM NaCl, 1 mM DTT, 1 mM PMSF

587 and $10 \%$ glycerol) for 16 hours at $4{ }^{\circ} \mathrm{C}$ with slow stirring. Protein inhibitor

588 cocktail (Sigma-Aldrich) was added to a final concentration of $1.5 x$. Protein

589 concentration was measured using the Pierce BCA Protein Assay Kit (Thermo 
Westra et al.

590 Scientific) and adjusted to $4 \mu \mathrm{g} / \mu \mathrm{l}$.

591

592 The standard binding reaction contained $2 \mu \mathrm{l}$ of 10x Binding Buffer (100 mM Tris

$593 \mathrm{pH} 7.5,500 \mathrm{mM} \mathrm{KCl}$ and $10 \mathrm{mM}$ DTT), 2.5\% glycerol, $5 \mathrm{mM} \mathrm{MgCl}$, 0.05\% NP-

594 40, 50 ng Poly (dl:dC), 20 fmol biotin-labeled probe and $16 \mu \mathrm{g}$ nuclear extract in

595 a final volume of $20 \mu \mathrm{l}$. For competition experiments, a 200-fold molar excess (4

596 pmol) of unlabeled probe was added.

598 Binding reactions were incubated at room temperature for $30 \mathrm{~min}$ and loaded

599 onto a $6 \%$ polyacrylamide $0.5 x$ TBE gel. After sample electrophoresis and

600 transfer to a nylon membrane, transferred DNA was crosslinked for 10 min and

601 the migration of the biotinylated probes and their complexes was detected by

602 chemiluminescence followed by film exposure.

604 Luciferase reporter assay

605 The double stranded oligonucleotide containing the SNP of interest (obtained as

606 described above) was cloned downstream the luciferase gene in the luciferase

607 reporter vector pGL3 promoter (Promega). For that, unlabeled double stranded

608 oligonucleotides containing the rs117701653, rs35926684 or rs34552516 were

609 amplified with specific primers containing the BamHI restriction site obtained 
Westra et al.

610 from IDT (Supplementary Table 19). The PCR was carried out in $50 \mu$ reaction

611 volume under the following program: $94^{\circ} \mathrm{C} 3 \mathrm{~min} ; 10$ cycles $94^{\circ} \mathrm{C} 30 \mathrm{sec}, 60^{\circ} \mathrm{C}$

$61240 \mathrm{sec}, 68^{\circ} \mathrm{C} 30 \mathrm{sec} ; 15$ cycles $94^{\circ} \mathrm{C} 30 \mathrm{sec}, 60^{\circ} \mathrm{C} 40 \mathrm{sec}, 68^{\circ} \mathrm{C} 30 \mathrm{sec} ; 72^{\circ} \mathrm{C} 10$

613 min. Both PCR products and pGL3 promoter vector were digested with BamHI

614 (New England Biolabs) for $1 \mathrm{~h}$ at $37^{\circ} \mathrm{C}$ and linearized vector was then

615 dephosphorylated for 30 min at $37^{\circ} \mathrm{C}$ with the Quick Dephosphorylation kit (New

616 England Biolabs). Digestion products were analyzed by electrophoresis in 1.2\%

617 agarose gels, and purified with QIAquick Gel Extraction Kit (Qiagen). Ligation of

618 SNP containing fragments into the pGL3 promoter plasmid was performed in a

619 ratio 1:50 (vector:insert) with T4 DNA ligase at $16^{\circ} \mathrm{C}$ overnight and transformed

620 into JM109 competent cells (Promega). Plasmids from independent colonies

621 were isolated using Wizard Plus SV minipreps DNA purification system

622 (Promega) and sequenced using RV primer 4 (Promega) by Eton Bioscience. For

623 each of the SNP, 3 colonies harboring the SNP-construct cloned "in sense" in

624 the pGL3 promoter vector were selected for further plasmid isolation for

625 transfection into Jurkat T cells.

627 Three independent transfection experiments for each construct were performed, 628 each in duplicate. $0.6 \times 10^{4}$ Jurkat cells in $0.1 \mathrm{ml}$ of Opti-MEM (Gibco) were

629 transfected with $0.8 \mu \mathrm{g}$ of pGL3-promoter vectors, either without insert or with

630 any of the six SNP-containing inserts, along with $0.2 \mu \mathrm{g}$ of pRL-TK Renilla

631 luciferase vector (Promega) using $1.5 \mu$ of Lipofectamine LTX Reagent and $1 \mu \mathrm{l}$ 
Westra et al.

632 of PLUS Reagent (both from Invitrogen). After 16 hours of transfection,

633 luciferase activity was measured using the Dual-Glo Luciferase assay system

634 (Promega) following manufacturer instructions. Firefly luciferase activity was

635 expressed as relative luciferase units (RLU) after correction for Renilla luciferase

636 activity to adjust for transfection efficiency. Data were normalized to those cells

637 transfected with empty pGL3-promoter vector. Results from the different clones

638 were pooled together and expression levels compared by unpaired two-sided t639 test. 
Westra et al.

640 Figure and Table Captions

\section{Table 1}

642 Overview of causal variants in selected loci. * identified using lower MAF

643 threshold of 0.005 . Greyed out posteriors are not significant in the primary

644 association analysis. Functional annotations: 1 DNAse1, 2 H3K3me3, 3

645 ChromHMM Enhancers, 4 ChromHMM Promoters, 5 Haploreg Alters motif, 6

646 Haploreg alters binding, 7 ATAC-seq, 8 eQTL T cells, 9 eQTL whole blood. n.s.:

647 non-specific binding. 


\begin{tabular}{|c|c|c|c|c|c|c|c|c|c|c|c|c|}
\hline \multicolumn{4}{|c|}{ Causal in } & \multicolumn{4}{|c|}{ Posterior } & \multirow[b]{2}{*}{ Variant Type } & \multirow[b]{2}{*}{ Non-coding } & \multirow[b]{2}{*}{$\begin{array}{l}\text { Functional } \\
\text { Annotation }\end{array}$} & \multicolumn{2}{|c|}{ Functional evidence } \\
\hline RA & T1D & Locus & Variant & $\begin{array}{l}\text { Type of } \\
\text { association }\end{array}$ & RA & T1D & Combined & & & & EMSA & Luciferase assay \\
\hline \multirow[t]{2}{*}{$\mathrm{x}$} & & DNASE1L3 & rs35677470 & Primary & 0.81 & & & R206C & & $3,5,8,9$ & & \\
\hline & & CD28/CTLA4 & rs3087243 & Primary (T1D) & 0.01 & 0.46 & 0.91 & & $x$ & $2,3,6$ & n.s. & \\
\hline $\mathrm{x}$ & & & rs 117701653 & Primary (RA) & 0.82 & & 0.00 & & $\mathrm{x}$ & $1,2,3,5,7$ & $\mathrm{C}>\mathrm{A}$ & $\begin{array}{l}A>\text { control }(p=0.0024), C>A \\
(p=0.0017)\end{array}$ \\
\hline$x$ & & & rs55686954 & Primary (RA) & 0.14 & & & & $x$ & $2,3,4$ & $A>G$ & n.s. \\
\hline \multirow[t]{2}{*}{$\mathrm{x}$} & & TNFAIP3 & rs35926684 & Primary & 0.24 & & 0.11 & Indel & $x$ & 3,5 & $G A>G$ & $\begin{array}{l}G>\text { control }(p=0.0124), \\
G A>G(p=0.0533)\end{array}$ \\
\hline & $\mathrm{x}$ & MEG3 & rs34552516 & Primary & & 0.37 & & Indel & $x$ & 3,5 & $\mathrm{TC}>\mathrm{T}$ & $\begin{array}{l}T>\text { control }(p=0.039), T C>T \\
(p=0.0454)\end{array}$ \\
\hline$x$ & $x$ & PTPN22 & rs2476601 & Primary & 0.52 & 0.92 & 0.90 & R620W & & $5,6,8,9$ & & \\
\hline$x$ & $\mathrm{x}$ & TYK2 & rs 34536443 * & Primary & 0.97 & 0.98 & 1.00 & P1104A & & 5,9 & & \\
\hline \multirow[t]{7}{*}{$\mathrm{x}$} & & & rs 35018800 * & Secondary (RA) & & & & A928V & & 5,6 & & \\
\hline & $\mathrm{x}$ & & rs12720356 * & Secondary (Cor & & & & $1684 S$ & & & & \\
\hline & $\mathrm{x}$ & SH2B3 & rs3184504 & Primary & & 0.33 & & R262W & & 5,9 & & \\
\hline & & ANKRD55 & rs11377254 & Primary & 0.88 & & 0.85 & Indel & $\mathrm{x}$ & $1,2,3,4,5,6,7$ & n.s. & \\
\hline & & REL/PUS10 & rs35149334 & Primary & 0.94 & & & & $\mathrm{x}$ & 5 & n.s. & \\
\hline & & IL2RA & rs61839660 & Primary & & 0.85 & & & $x$ & $1,2,3,5,6,7,9$ & n.s. & \\
\hline & $x$ & SIRPG & rs6043409 & Primary & & 0.25 & & V263A & & $5,8,9$ & & \\
\hline
\end{tabular}

1 DNAse1, 2 H3K3me3, 3 ChromHMM Enhancers, 4 ChromHMM Promoters, 5 Haploreg Alters motif, 6 Haploreg alters binding, 7 Atac-seq, 8 eQTL T-cells, 9 eQTL whole blood 
Westra et al.

\section{$648 \quad$ Figure 1}

649 We imputed our datasets with different reference panels: the European

650 subpopulation of 1000 genomes (EUR), full 1000 genomes (COSMO), and the

651 Haplotype Reference Consortium (HRC). A) We sequenced $7991 \mathrm{~kb}$ regions in

652568 individuals with ImmunoChip genotypes, and called 1,862 common

653 (MAF>1\%) variants. Imputation with COSMO and EUR recovers $96 \%$ of these

654 variants, while HRC imputation recovers 90\%. We calculated imputation

655 accuracy by correlating imputed genotypes with genotypes called from the

656 sequencing experiment (genomic $\left.r^{2}\right)$. At $r^{2}>0.5$, COSMO and EUR recover 93\%

657 of variants, while HRC recovers $86 \%$. B) Imputation quality scores (INFO) for

658 each reference panel in the RA dataset. COSMO shows highest increase in

659 number of variants (MAF>1\%) after imputation (2.5x; INFO>0.3) compared to

660 EUR (2.3x) and HRC (1.9x). 
Figure 1

A

Coverage above

B

Total coverage threshold

Coverage increase

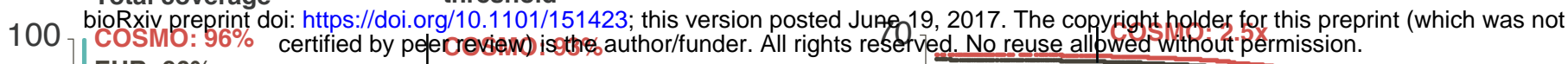
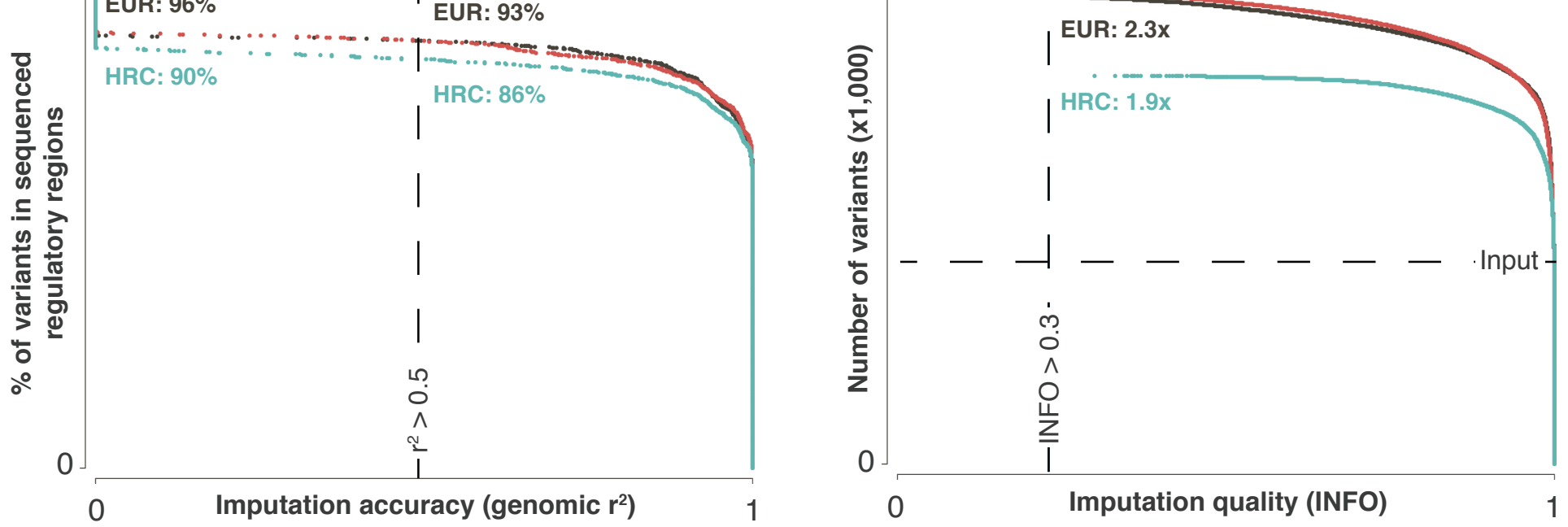
Westra et al.

\section{Figure 2}

662 We used the approximate Bayesian factor to determine $90 \%$ credible sets within

663 significant loci. A) A number of loci have a shared signal between diseases.

664 Inner ring of dots indicates whether locus has $\leq 10$ variants in credible set and

665 has a significant association signal, and is open otherwise. Middle ring shows

666 variants in each credible set. Highlighted segments indicate loci with causal

667 variant. Color intensity indicates posterior probability and grey when not

668 significant. Outer ring shows indel, promoter and missense coding annotation

669 for each variant in credible set. B) We are able to narrow down the list of causal

670 variants 5 in 8 out of 20 significant RA loci, and 11 out of 36 significant T1D loci.

671 For both diseases, we find two loci that are explained by a single variant. C)

672 From the credible sets, we defined groups of interesting loci, based on the

673 presence of a high posterior missense variant $(>0.2)$, indel $(>0.2)$ or SNP $(>0.8)$.

674 We applied several follow-up analyses to these loci, including conditional

675 analysis, exhaustive pairwise and haplotype analysis when a secondary signal

676 was present, and functional analysis (EMSA) for non-coding loci. 


\section{Figure 2}

A
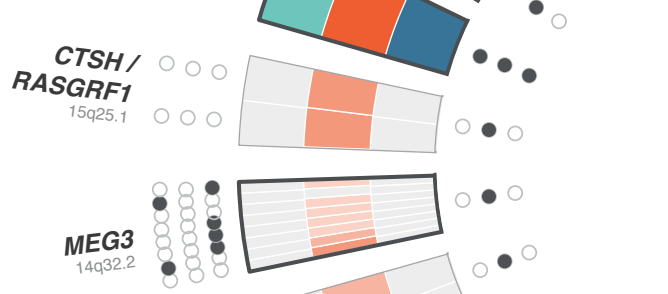
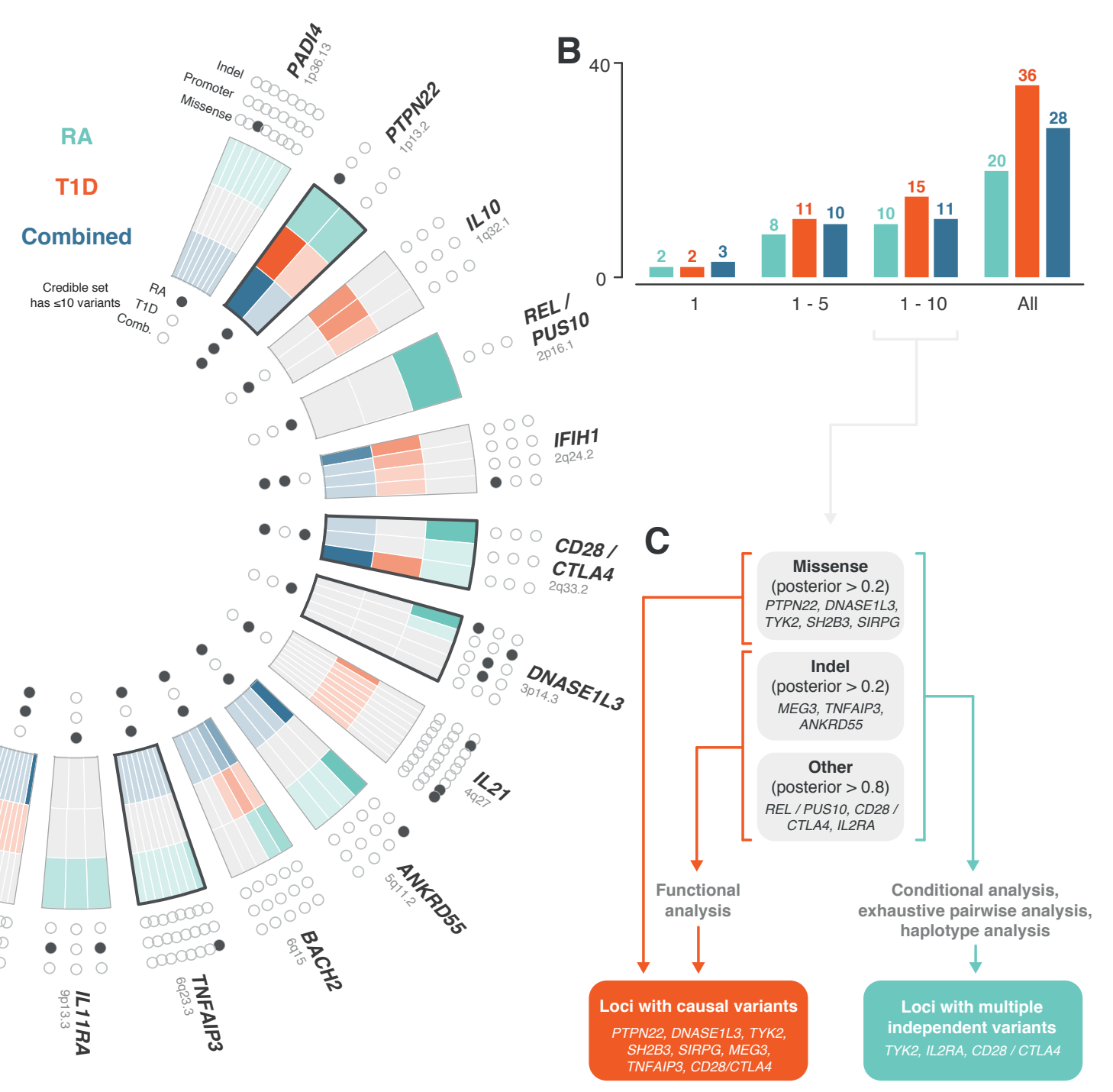

RA

\section{\begin{tabular}{l|l|l}
\hline$\square$ \\
\hline
\end{tabular}}

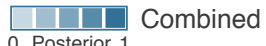

Not associated 
Westra et al.

\section{$677 \quad$ Figure 3}

678 Analysis in the CD28/CTLA4 locus. A) The regional association plot for the

679 combined analysis shows a single variant (rs3087243), near CTLA4, in the

680 credible set. Conditioning on rs30872043 reveals rs117701653 as an

681 independent association. Color indicates LD with top-associated variant. Square

682 indicates presence in credible set. B) Exhaustive pairwise analysis shows that

683 rs3087243+rsrs117701653 pair has strongest association. Green color indicates

684 -Log10(pairwise p-value), purple color indicates pairwise LD. C) Haplotype

685 analysis using rs30872043 andrs11701653, using the AG haplotype as

686 reference. The $\mathrm{C}$ allele of rs117701653 shows largest decrease in risk in RA, and

687 the A of rs30872043 in T1D. D) EMSA using probes for rs117701653 and

688 rs3087243 as a functional follow-up in Jurkat T cells. We observe an extra band

689 in the lane with protein sample and biotin probe for the C-allele that is not

690 observed for the other probes. The band disappears when adding non-labeled

691 probe, suggesting competition between labeled and non-labeled probe for

692 binding protein. E) Luciferase assay for rs117701653 using pGL3 plasmids in

693 Jurkat T cells. We calculated relative luciferase activity units (RLU) using the

694 activity of the empty plasmid (pGL3) as reference, and observed significant

695 increase in luciferase activity for the A allele, and a further significant increase in

696 luciferase activity for the $\mathrm{C}$ allele, which verifies that both alleles affect protein

697 binding, albeit likely with different affinities. 
bioRxiv preprint doi: https://doi.org/10.1101/151423; this version posted June 19, 2017. The copyright holder for this preprint (which was not

Figure 3 - CD28\%"CTL'A4"

A RA, T1D Combined
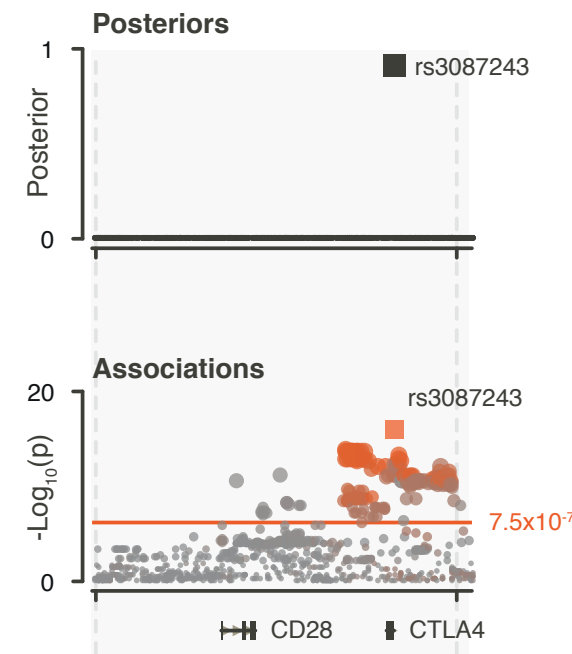

$204.45 \quad$ Chr $2(\mathrm{mb})$
$\begin{gathered}\text { Linkage } \\ \text { Disequilibrium }\end{gathered}$

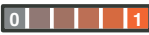

C

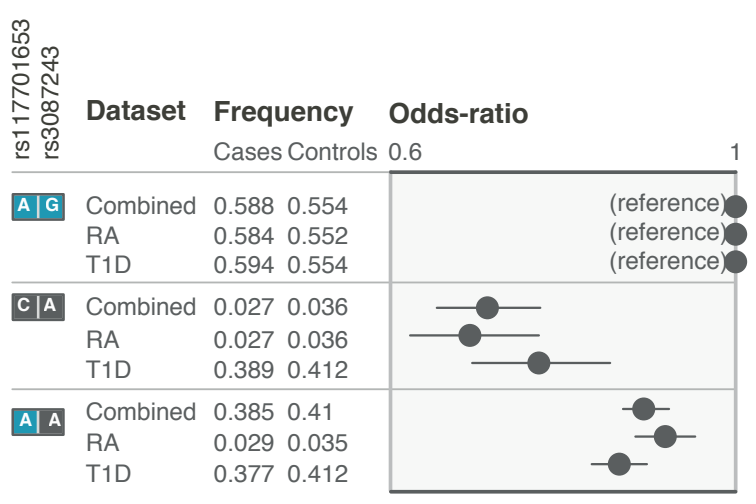

B Rheumatoid Arthritis

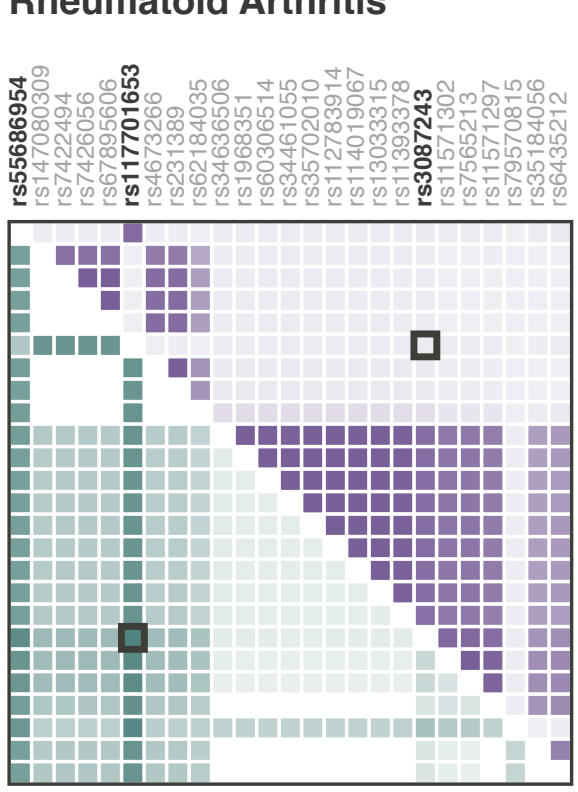

$-\log 10(p-v a l u e)$

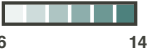

Linkage Disequilibrium \begin{tabular}{|l|l|}
\hline 1 \\
\hline
\end{tabular}

rs55686954 rs55686954

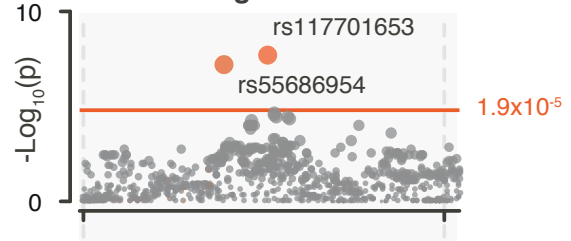

Conditioning on rs3087243,

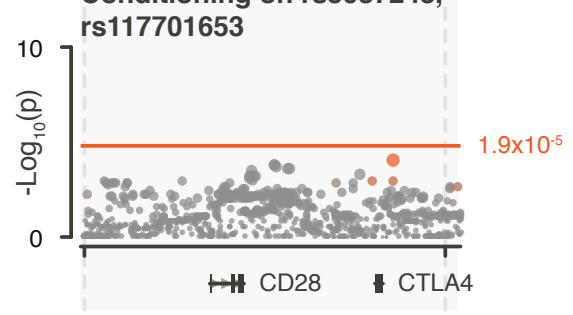

$204.45 \quad$ Chr $2(\mathrm{mb}) \quad 204.8$ rs3087243 + rs 117701653: $\log 10(p)=13.81$ Most significant pair out of 567,629

E

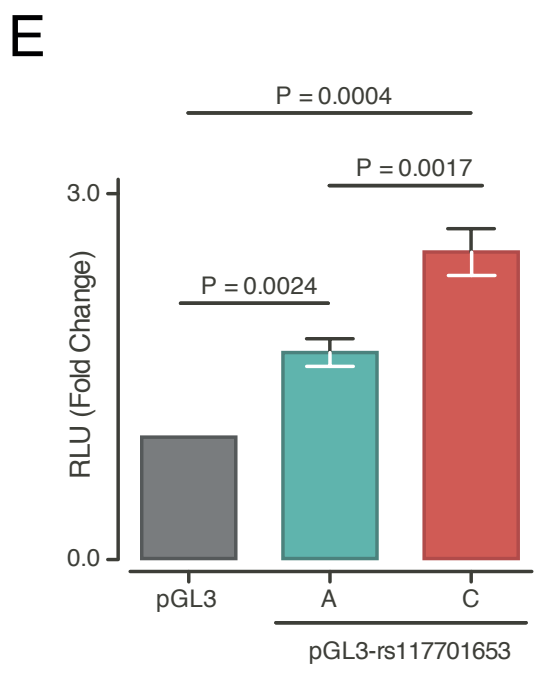

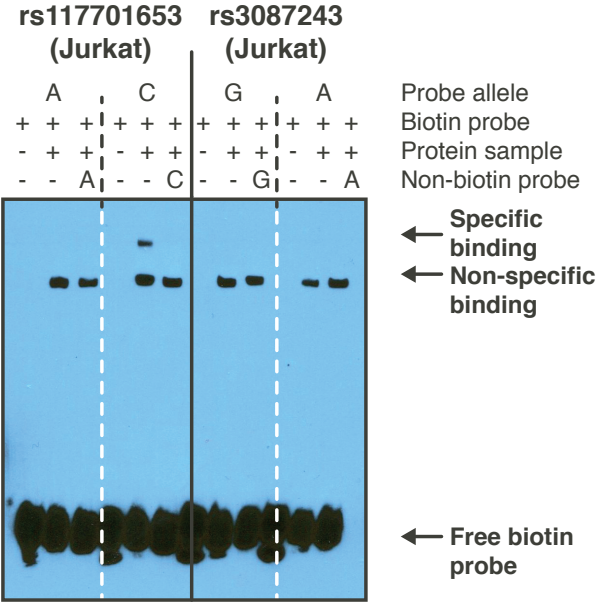


Westra et al.

\section{$698 \quad$ Figure 4}

699 Analysis in the TNFAIP3 locus. A) The variant with the strongest posterior in this 700 locus is rs35926684, a G/GA indel, associated with RA. Conditional on

701 rs35926684, we observe a significant secondary association with rs58721818.

702 B) Exhaustive pairwise association analysis in RA indicates that there are 6 pairs 703 with a lower p-value than rs35926684+rs58721818, although the top-associated 704 pair (rs69220220+rs58721818) has an equivalent $p$-value $\left(-\log _{10}(p)=13.94\right.$ vs 705 14.21). C) Haplotype analysis with rs35926684+rs58721818, and previously 706 reported variants rs6920220 and rs5029937 shows that rs35926684 and 707 previously reported top variant rs6920220 are often located on the same 708 haplotype (GAGC), although a rare haplotype exists with only the alternative 709 allele of rs35926684, which causes a similar increase in risk, although with larger 710 standard error. D) EMSA analysis using a G and GA probe for rs35926684. We

711 observe an extra band in the lane with protein sample and biotin probe for the

712 GA-allele that is not observed for the other probes. The band disappears when

713 adding non-labeled probe, suggesting competition between labeled and non-

714 labeled probe for binding protein. E) Luciferase assay for rs35926684 shows

715 that both $\mathrm{G}$ and GA consequently alter luciferase expression. 
bioRxiv preprint doi: https://doi.org/10.1101/151423; this version posted June 19, 2017. The copyright holder for this preprint (which was not

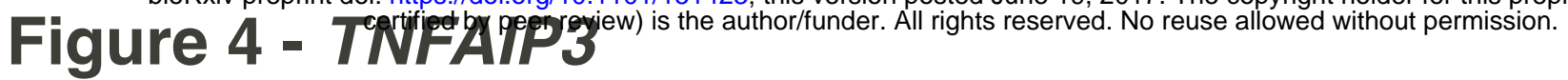

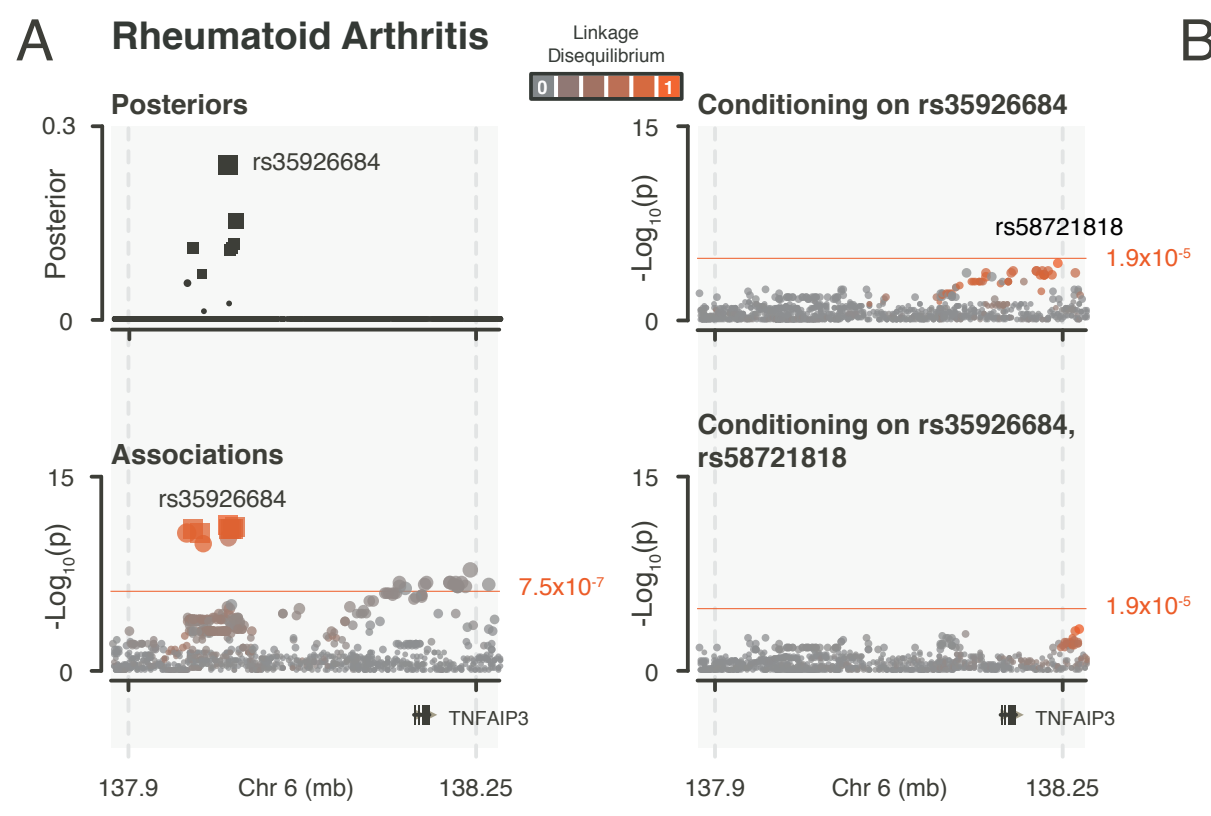

C

\begin{tabular}{|c|c|c|}
\hline & Frequ & ency \\
\hline 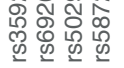 & Cases & Controls \\
\hline $\mathrm{G}|\mathrm{G}| \mathrm{G} \mid \mathrm{C}$ & 0.743 & 0.771 \\
\hline G|G|G| & 0.014 & 0.011 \\
\hline \begin{tabular}{l|l|l|l|}
$G|A| G \mid$ \\
\end{tabular} & 0.217 & 0.198 \\
\hline 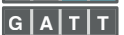 & 0.026 & 0.02 \\
\hline
\end{tabular}

Odds-ratio

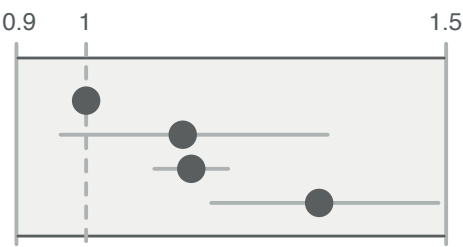

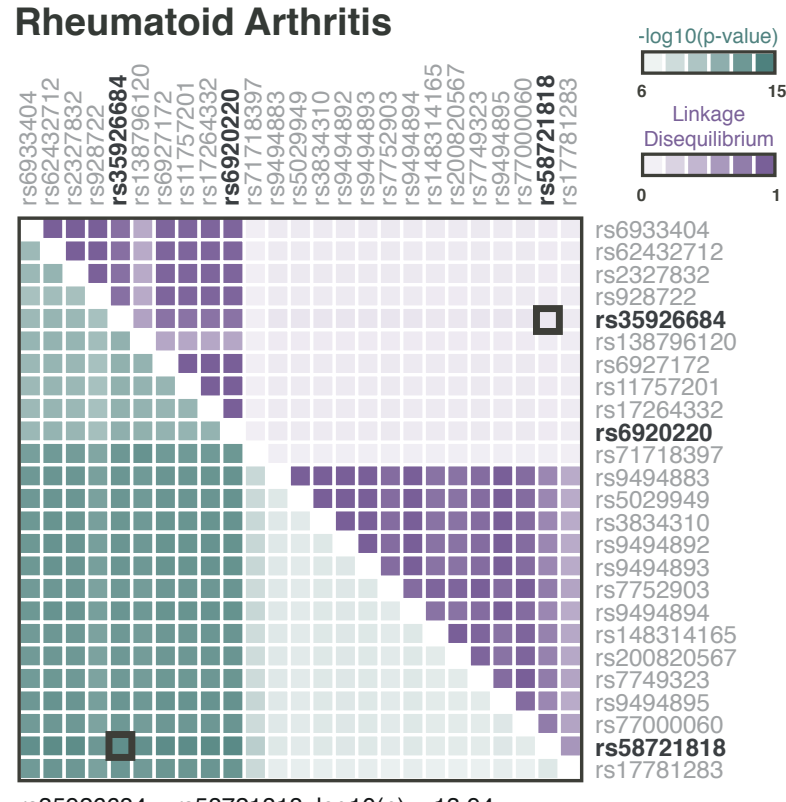

rs35926684 + rs58721818, $\log 10(p)=13.94$

rs35926684 + rs58721818 is the seventh most significant pair of 697,970 pairs; Top pair: $r s 6920220+r s 58721818, \log 10(p)=14.21$

$D \begin{gathered}\text { rs35926684 } \\ \text { (Jurkat) }\end{gathered}$

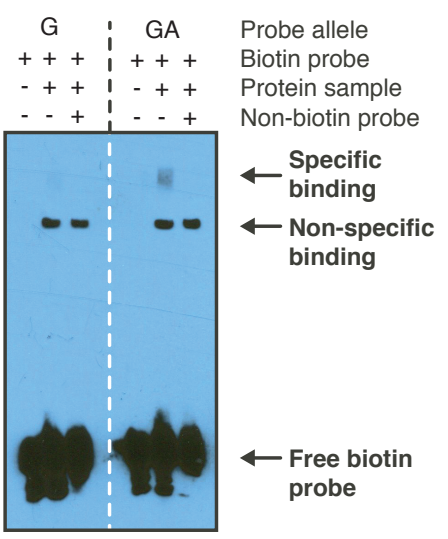

$E$

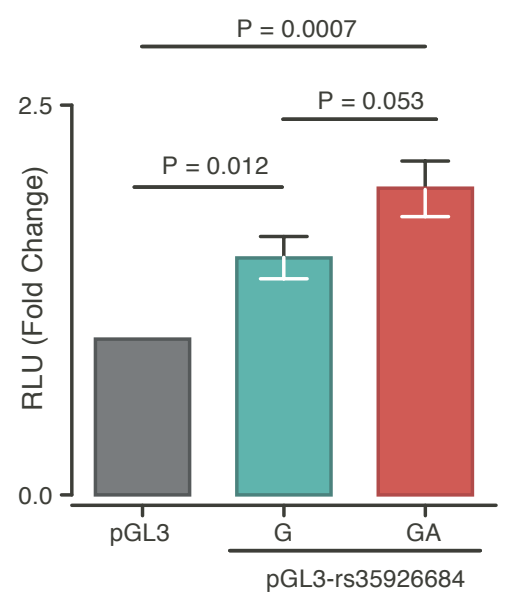


Westra et al.

\section{$716 \quad$ Figure 5}

717 Analysis in the MEG3 locus. A) Region plot for the MEG3 locus in T1D. We

718 observe two variants in the credible set (rs56994090 and indel rs34552516;

719 indicated by squares). We did not observe secondary signals when conditioning

720 on rs56994090. B) EMSA analysis for rs354552516 and rs56994090. Left: the

721 TC allele of rs34552516 shows a band that disappears when adding non-labeled

722 TC probe as competitor, suggesting specific binding. C) Consequently, a

723 luciferase assay for rs34552516 shows an increase of luciferase activity for the

724 TC allele relative to the T allele and empty vector. 
bioRxiv preprint doi: https://doi.org/10.1101/151423; this version posted June 19, 2017. The copyright holder for this preprint (which was not

Figure $5-M E G 3^{\circ}$

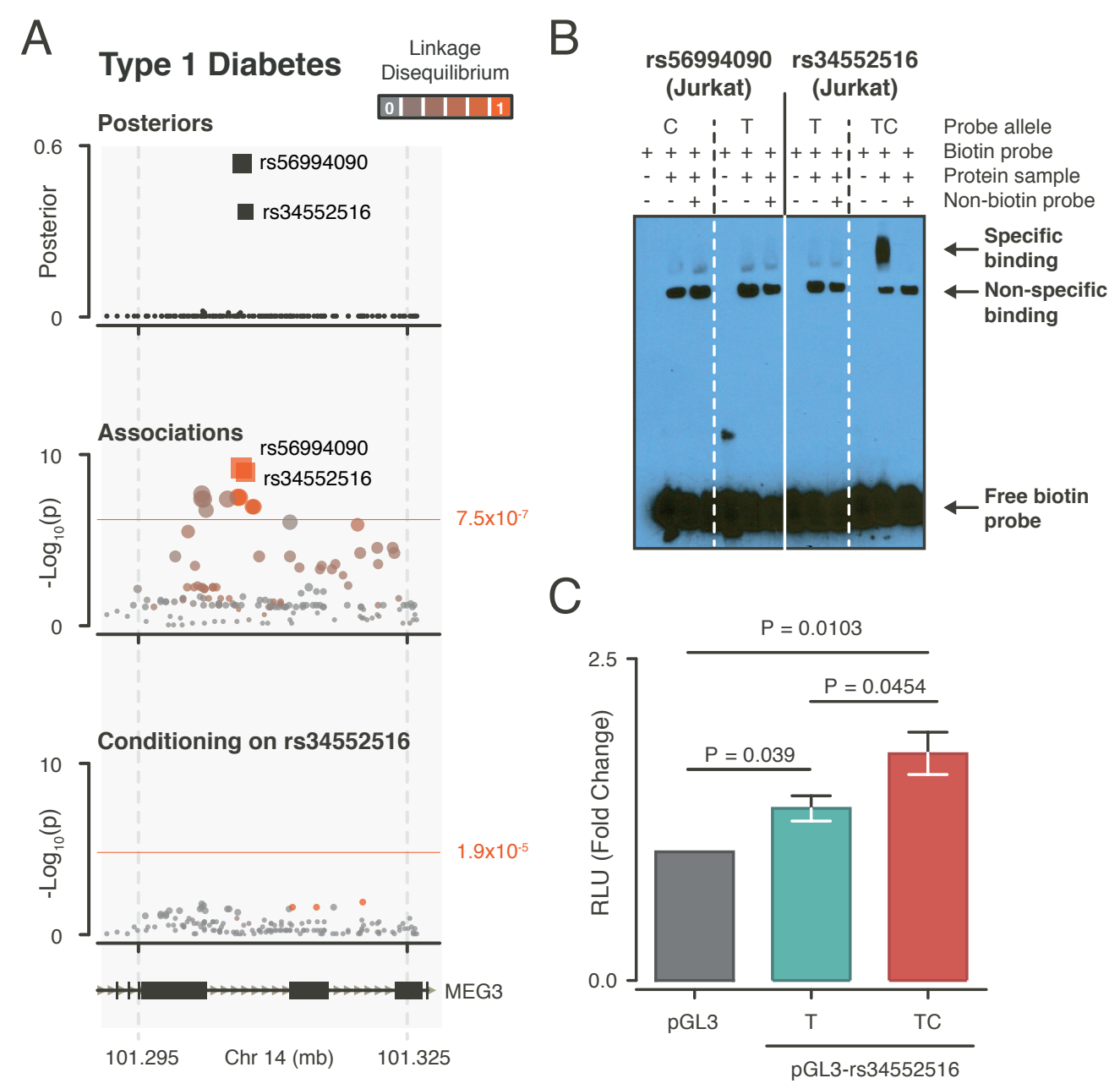


Westra et al.

\section{Supplementary Figures}

\section{Supplementary Figure 1}

727 A) Out of the 902 sequenced regions, 799 had $>20 x$ coverage at $80 \%$ of

728 sequenced bases in at least $50 \%$ of the samples. B) 87 samples had less than

$72920 x$ coverage in at least $90 \%$ of the sequences bases. C) The 1,170 variants out

730 of the 1,862 called variants that overlapped within 568 ImmunoChip genotyped

731 individuals were highly correlated for both RA and T1D

\section{Supplementary Figure 2}

734 Top: Imputation quality (INFO) scores for the RA and T1D datasets, imputed with

735 the European subpopulation of 1000 genomes (EUR), full 1000 genomes

736 (COSMO), and the Haplotype Reference Consortium (HRC) reference panels. In

737 T1D, HRC imputation was performed in three ways: using EAGLE (HRC /

738 EAGLE) or SHAPEIT (HRC / SHAPEIT) for phasing on the Sanger Institute

739 imputation server, or using EAGLE for phasing and imputation on the Michigan

740 imputation server (HRC / EAGLE / MICHIGAN). From left to right: comparing

741 variants with $\mathrm{MAF}>1 \%$, comparing variants with $\mathrm{MAF}>1 \%$ but excluding indels,

742 and comparing all variants. For MAF>1\% variants, COSMO outperforms both

743 EUR and HRC. 
Westra et al.

\section{Supplementary Figure 3}

747 Imputation accuracy (genomic $r^{2}$ ) for the RA and T1D datasets, imputed with the 748 European subpopulation of 1000 genomes (EUR), full 1000 genomes (COSMO), 749 and the Haplotype Reference Consortium (HRC) reference panels. Genomic r2 750 was calculated by correlating imputed dosages with sequenced variants for the 751 same individuals. In T1D, HRC imputation was performed in three ways: using 752 EAGLE (HRC / EAGLE) or SHAPEIT (HRC / SHAPEIT) for phasing on the Sanger 753 Institute imputation server, or using EAGLE for phasing and imputation on the 754 Michigan imputation server (HRC / EAGLE / MICHIGAN). From left to right: 755 comparing variants with MAF>1\%, comparing variants with MAF>1\% but 756 excluding indels, and comparing all variants. In all cases, COSMO outperforms 757 both EUR and HRC.

759 Supplementary Figure 4

760 We compared imputation quality (INFO score) with imputation accuracy

761 (genomic $r^{2}$ ) in the T1D dataset, and observed a strong correlation $\left(r^{2}=0.82\right)$.

\section{Supplementary Figure 5}

764 In the T1D dataset, 72 variants (MAF>1\%) that were present in our gold 765 standard genotype dataset were not present after imputation. A) The majority $766(69 \%)$ of these variants were indels and B) variants of low allele frequency (44\% 
Westra et al.

767 with $\mathrm{MAF}<5 \%$ ). C) For those variants with a low $\mathrm{MAF}(\mathrm{MAF}<1 \%)$, or with a low

768 correlation with gold standard genotypes $(r 2<0.5)$, the majority $(77 \%)$ were low

769 frequency variants.

771 Supplementary Figure 6

772 In $66 \%$ of the 76 tested loci, the association statistics (Z-scores) between RA

773 and T1D are positively correlated.

775 Supplementary Figure 7

776 Region plot for the PTPN22 locus. The credible set consists two variants

777 (indicated by squares): we observe two significant $\left(\mathrm{p}<7.5 \times 10^{-7}\right)$ associations in

778 RA and T1D. These associations include rs2476601, which causes a R620W

779 coding change in the PTPN22 protein and has a high posterior $(0.78)$ in the

780 combined analysis. No significant secondary signals are observed when

781 conditioning on rs2476601. Color indicates LD between top associated variant.

783 Supplementary Figure 8

784 A) Region plot for the TYK2 locus. Considering previous analysis in this region, 785 we decreased the MAF threshold for this region to $0.5 \%$. For each analysis, the 786 credible set consists of a single variant, rs34536443, causing a P1104A change in 787 TYK2. Conditional on P1104A, we observe a secondary association from rs35018800 in 
Westra et al.

788 RA, causing a A928V change in TYK2. Further conditioning indicates a tertiary 789 association from rs 12720356 in the combined analysis, causing a 16845 change in 790 TYK2. Finally, conditional on these three coding variants, we observe a quaternary 791 association from rs35074907. B) Top 25 SNPs as identified by pairwise exhaustive 792 analysis. In RA and the combined analysis, rs34536443+rs35018800 is the top 793 associated pair. In T1D, however, there are 138 pairs with a lower $p$-value, with 794 rs35018800 + rs12720356 having the strongest association. C) Haplotype analysis 795 using rs34536443, rs12720356, rs35018800, and rs35074907 using the GGAG

796 haplotype as a reference. All haplotypes confer independent relative risk reduction, 797 except for GGAA, which increases risk in T1D, relative to the reference haplotype.

800 Region plot for the SH2B3 locus. The credible set for T1D contains two variants, 801 including rs3184504, causing a R262W change in SH2B3. Conditioning on 802 rs3184504, we do not observe a secondary association.

804 Supplementary Figure 10

805 Region plot for the DNASE1L3 locus. The credible set consists of two variants in 806 RA, including rs35677470, causing a R206C coding change in the DNASE1L3 807 protein. No significant secondary signals are observed when conditioning on 808 rs35677470. 
Westra et al.

\section{Supplementary Figure 11}

811 Region plot for the SIRPG locus. The credible set consists of seven variants in

812 T1D, including rs6043409, causing a V263A coding change in the SIRPG

813 protein. No significant secondary signals are observed when conditioning on

814 rs6043409.

\section{Supplementary Figure 12}

817 Region plot for the ANKRD55 locus. We did not observe a significant

818 association for T1D, while for RA, the credible set contained two variants:

819 rs11377254 and rs7731626 (indicated by squares). No secondary signals were

820 observed when conditioning on rs11377254.

\section{Supplementary Figure 13}

823 Region plot for the REL locus. The credible set for RA contains a single variant

824 with strong posterior (rs35149334), but shows no association in T1D.

825 Conditioning on rs35149334, we do not observe a secondary association.

\section{Supplementary Figure 14}

828 Region plot for the IL2RA locus. The credible set for T1D contains two variants, 829 with rs61839660 having the largest posterior (0.85). When performing 
Westra et al.

830 conditional analysis, a secondary association is observed from rs4747846, a

831 tertiary association from rs41295159, and finally, a quaternary association from

832 rs704778. B) Pairwise exhaustive analysis in T1D shows that there are 0 pairs

833 with a lower association p-value than rs61839660 + rs474846. C) Haplotype

834 analysis suggests independent and opposite effects from haplotypes carrying

835 rs681839660 and rs706778 alternate alleles.

837 Supplementary Figure 15

838 A) Region plots for the CD28/CTLA4 locus: rs3087243, near CTLA4, has an

839 increased posterior in the combined analysis compared with T1D, indicating a

840 shared effect. In RA, rs117701653, near CD28, has the highest posterior. Both

841 rs3087243 and rs11701653 are independently associated with RA, but not T1D.

842 B) Exhaustive pairwise analysis for RA shows that the rs117701653+rs3087243

843 pair has the strongest association for RA, but not T1D. C) Left to right: specific

844 band in EMSA for rs117701653 C allele can be competed away using non-

845 labeled A probe, indicating specific binding for A allele as well. Dose titration of

846 labeled $\mathrm{C}$ and $\mathrm{A}$ allele probes (quantities in fmol) indicates that $\mathrm{A}$ allele also

847 shows allele specific binding at higher probe quantities. EMSA in THP-1

848 monocyte cells does not show band for specific binding that is visible in Jurkat

849 T cells for the rs117701653 C allele. EMSA for rs55686954 shows allele specific

850 binding for the A allele. D) When performing a luciferase assay on rs117701653 
Westra et al.

851 and rs55686954, we observe allele specific enhancer activity for rs 117701653

852 but not rs55686954.

\section{Supplementary Figure 16}

855 Promoter capture hi-C plots for the CD28/CTLA4, TNAIP3 and MEG3 loci show

856 multiple contacts between bait sequences containing potential causal variants

857 and downstream genomic regions. Figures adapted from http://www.chicp.org/

859 Supplementary Figure 17

860 Region plot for the TNFAIP3 locus showing (from top to bottom) genes,

861 posterior probabilities, and association p-values. The credible set for RA

862 contains 8 variants, including indel rs35926684 (indicated by squares). No

863 significant association was observed for T1D. When conditioning on

864 rs35926684, a suggestive secondary signal was observed from rs58721818. B)

865 Exhaustive pairwise testing shows that there are 6 pairs having a stronger

866 association with RA than rs35926684 + rs58721818, with rs6920220 +

867 rs58721818 showing the strongest association. C) Left to right: EMSA dose

868 titration of labeled G and GA allele probes for rs355926684 (quantities in fmol)

869 indicates that $\mathrm{G}$ allele also shows allele specific binding at higher probe

870 quantities. Specific binding for the GA allele is not observed in THP-1 monocyte

871 cells. EMSA in Jurkat cells for rs6920220 does not indicate specific binding. 
Westra et al.

872

873 Supplementary Figure 18

874 A) Region plot for the MEG3 locus showing (from top to bottom) genes,

875 posterior probabilities, and association p-values. We observe two variants in the

876 credible set (rs56994090 and indel rs34552516; indicated by squares) for T1D,

877 but no association in RA. We did not observe secondary signals when

878 conditioning on rs56994090. B) EMSA in Jurkat T cells and THP-1 monocyte

879 cells, shows no specific binding for the TC allele of rs34552516.

881 Supplementary Tables

882 Supplementary Table 1

883 Overview of the cases and controls for each of the cohorts included in this 884 study.

886 Supplementary Table 2

887 List of ImmunoChip regions, and regions with significant associations with RA or 888 T1D published in previous studies.

890 Supplementary Table 3

891 Statistics for variants called from targeted sequencing experiment (MAF > 1\%). 
Westra et al.

\section{Supplementary Table 4}

894 Imputation accuracy as determined by correlating imputed genotype dosages

895 with genotypes called from targeted sequencing experiment for variants that are 896 both present and absent on ImmunoChip.

\section{Supplementary Table 5}

899 Differences between imputation reference panels, by testing the difference in 900 imputation accuracy (t-test).

\section{Supplementary Table 6}

903 Number of variants used as input for imputation, and output of imputation, at 904 different imputation quality (INFO) score and allele frequency thresholds for each 905 imputation reference panel.

\section{Supplementary Table 7}

908 Comparison of results presented in Okada et al. with the RA association

909 analysis, for regions significant in this study. For each study, we compared the

910 strongest association per region. Gt: genotyped variant. 
Westra et al.

\section{Supplementary Table 8}

913 Comparison of results presented in Onengut-Gumuscu et al. with the T1D

914 association analysis, for regions significant in this study. For each study, we

915 compared the strongest association per region. Gt: genotyped variant.

\section{Supplementary Table 9}

918 Correlations between RA and T1D association statistic Z-scores for the 76

919 tested loci.

921 Supplementary Table 10

922 Comparison of results from the combined analysis with the RA association

923 analysis, for regions significant in this study. For each analysis, we compared

924 the association with the strongest association per region. Gt: genotyped variant.

926 Supplementary Table 11

927 Comparison of results from the combined analysis with the T1D association

928 analysis, for regions significant in this study. For each analysis, we compared

929 the association with the strongest association per region. Gt: genotyped variant. 
Westra et al.

$93290 \%$ credible sets identified in this study: association results for regions that

933 have $\leq 10$ variants in the $90 \%$ credible set and are significant in either RA, T1D,

934 or the combined analysis.

936 Supplementary Table 13

937 Conditional analysis results for the RA, T1D and combined analysis.

939 Supplementary Table 14

940 Haploreg annotations for candidate variants.

942 Supplementary Table 15

943 Overlap DNAse-I, H3K4me3, ChromHMM enhancers and ChromHMM

944 promoters for both immune cell type groups and other cell types.

946 Supplementary Table 16

947 Overlap of credible sets with ATAC-seq peaks called from time course

948 experiment in CD4+ T cells. 
bioRxiv preprint doi: https://doi.org/10.1101/151423; this version posted June 19, 2017. The copyright holder for this preprint (which was not certified by peer review) is the author/funder. All rights reserved. No reuse allowed without permission.

Westra et al.

951 eQTL overlap for the $90 \%$ credible sets in the RA, T1D, and combined analysis.

952

953 Supplementary Table 18

954 Oligonucleotide probes used during EMSA analysis.

955

956 Supplementary Table 19

957 Primers used for cloning Luciferase assay plasmids. 
Westra et al.

\section{References}

960 1. Maller, J. B. et al. Bayesian refinement of association signals for 14 loci in 961 3 common diseases. Nat. Genet. 44, 1294-301 (2012).

2. Wakefield, J. A Bayesian Measure of the Probability of False Discovery in Molecular Genetic Epidemiology Studies (DOI:10.1086/519024). Am. J. Hum. Genet. 83, 424 (2008).

3. Eyre, S. et al. High-density genetic mapping identifies new susceptibility loci for rheumatoid arthritis. Nat. Genet. 44, 1336-40 (2012).

4. Onengut-Gumuscu, S. et al. Fine mapping of type 1 diabetes susceptibility loci and evidence for colocalization of causal variants with lymphoid gene enhancers. Nat. Genet. 47, 381-386 (2015).

6. Palmer, J. P. et al. Insulin antibodies in insulin-dependent diabetics before insulin treatment. Science 222, 1337-9 (1983).

974 7. Baekkeskov, S. et al. Identificationof the $64 \mathrm{~K}$ autoantigen in insulindependent diabetes as the GABA-synthesizing enzyme glutamic acid decarboxylase. Nature 347, 151-156 (1990).

8. Okada, Y. et al. Genetics of rheumatoid arthritis contributes to biology and drug discovery. Nature 506, 376-81 (2014). 
Westra et al.

to single variant resolution. bioRxiv 28688 (2015). doi:10.1101/028688

10. Gaulton, K. J. et al. Genetic fine mapping and genomic annotation defines causal mechanisms at type 2 diabetes susceptibility loci. Nat. Genet. 47, 1415-1425 (2015)

11. Farh, K. K. et al. Genetic and epigenetic fine mapping of causal autoimmune disease variants. Nature 518, 337-343 (2015).

12. Trynka, G. et al. Chromatin marks identify critical cell types for fine mapping complex trait variants. Nat. Genet. 45, 124-30 (2013).

13. McCarthy, S. et al. A reference panel of 64,976 haplotypes for genotype imputation. Nat. Genet. 48, 1279-1283 (2016).

14. Abecasis, G. R. et al. A map of human genome variation from populationscale sequencing. Nature 467, 1061-73 (2010).

15. Begovich, A. B. et al. A missense single-nucleotide polymorphism in a gene encoding a protein tyrosine phosphatase (PTPN22) is associated with rheumatoid arthritis. Am. J. Hum. Genet. 75, 330-7 (2004).

16. Bottini, N. et al. A functional variant of lymphoid tyrosine phosphatase is associated with type I diabetes. Nat. Genet. 36, 337-338 (2004).

17. Diogo, D. et al. TYK2 protein-coding variants protect against rheumatoid arthritis and autoimmunity, with no evidence of major pleiotropic effects on non-autoimmune complex traits. PLoS One 10, e0122271 (2015). 
Westra et al.

1001

1002

1003

1004

1005

1006

1007

1008

1009

1010

1011

1012

1013

1014

1015

1016

1017

1018

1019

1020

1021

susceptibility variants identifies a novel association at DNASE1L3. Arthritis

Res. Ther. 16, (2014).

19. Al-Mayouf, S. M. et al. Loss-of-function variant in DNASE1L3 causes a familial form of systemic lupus erythematosus. Nat. Genet. 43, 1186-1188 (2011).

20. Ueki, M. et al. Caucasian-specific allele in non-synonymous single nucleotide polymorphisms of the gene encoding deoxyribonuclease I-like 3, potentially relevant to autoimmunity, produces an inactive enzyme. Clin. Chim. Acta 407, 20-24 (2009).

21. Fortune, M. D. et al. Statistical colocalization of genetic risk variants for related autoimmune diseases in the context of common controls. Nat. Genet. 47, 839-46 (2015).

22. Aguet, F. et al. Local genetic effects on gene expression across 44 human tissues. bioRxiv (2016).

23. Javierre, B. M. et al. Lineage-Specific Genome Architecture Links Enhancers and Non-coding Disease Variants to Target Gene Promoters. Cell 167, 1369-1384.e19 (2016).

24. Tsoi, L. C. et al. Identification of 15 new psoriasis susceptibility loci highlights the role of innate immunity. Nat. Genet. 44, 1341-1348 (2012).

25. Jostins, L. et al. Host-microbe interactions have shaped the genetic architecture of inflammatory bowel disease. Nature 491, 119-124 (2012). 
Westra et al.

1022 26. Beecham, A. H. et al. Analysis of immune-related loci identifies 48 new

1023 susceptibility variants for multiple sclerosis. Nat. Genet. 45, 1353-1360

1024 (2013).

1025 27. Lessard, C. J. et al. Variants at multiple loci implicated in both innate and adaptive immune responses are associated with Sjögren's syndrome. Nat. Genet. 45, 1284-1292 (2013).

28. Cordell, H. J. et al. International genome-wide meta-analysis identifies new primary biliary cirrhosis risk loci and targetable pathogenic pathways. Nat. Commun. 6, 8019 (2015).

1031 29. Bentham, J. et al. Genetic association analyses implicate aberrant regulation of innate and adaptive immunity genes in the pathogenesis of systemic lupus erythematosus. Nat. Genet. 47, 1457-1464 (2015).

30. Trynka, G. et al. Dense genotyping identifies and localizes multiple common and rare variant association signals in celiac disease. Nat. Genet. 43, 1193-201 (2011).

31. McGovern, A. et al. Capture Hi-C identifies a novel causal gene, IL20RA, in the pan-autoimmune genetic susceptibility region 6q23. Genome Biol.

32. Zhou, Y. et al. Activation of p53 by MEG3 Non-coding RNA. J. Biol. Chem. 282, 24731-24742 (2007). 17, 212 (2016).

33. Wallace, C. et al. The imprinted DLK1-MEG3 gene region on chromosome 
Westra et al.

1043

1044

1045

1046

1047

1048

1049

1050

1051

1052

1053

1054

1055

1056

1057

1058

1059

1060

1061

1062

1063

14q32.2 alters susceptibility to type 1 diabetes. Nat. Genet. 42, 68-71

(2010).

34. Purcell, S. et al. PLINK: a tool set for whole-genome association and population-based linkage analyses. Am. J. Hum. Genet. 81, 559-75 (2007).

35. Browning, B. L. \& Browning, S. R. Genotype Imputation with Millions of Reference Samples. Am. J. Hum. Genet. 98, 116-126 (2016).

36. Loh, P.-R. et al. Reference-based phasing using the Haplotype Reference Consortium panel. Nat. Genet. 48, 1443-1448 (2016).

37. Delaneau, O., Marchini, J. \& Zagury, J.-F. A linear complexity phasing method for thousands of genomes. Nat. Methods 9, 179-181 (2011).

38. Durbin, R. Efficient haplotype matching and storage using the positional Burrows-Wheeler transform (PBWT). Bioinformatics 30, 1266-72 (2014).

39. Fuchsberger, C., Abecasis, G. R. \& Hinds, D. A. minimac2: faster genotype imputation. Bioinformatics 31, 782-4 (2015).

40. Li, H. \& Durbin, R. Fast and accurate short read alignment with BurrowsWheeler transform. Bioinformatics 25, 1754-60 (2009).

41. Van der Auwera, G. A. et al. From FastQ data to high confidence variant calls: the Genome Analysis Toolkit best practices pipeline. Curr. Protoc. Bioinformatics 43, 11.10.1-33 (2013).

42. Powell, J. E., Visscher, P. M. \& Goddard, M. E. Reconciling the analysis of 
Westra et al.

1064

1065

1066

1067

1068

1069

1070

1071

1072

1073

1074

1075

1076

1077

1078

1079

1080

1081

1082

1083

IBD and IBS in complex trait studies. Nat. Rev. Genet. 11, 800-805 (2010).

43. Zhernakova, D. V et al. Identification of context-dependent expression quantitative trait loci in whole blood. Nat. Genet. (2016). doi:10.1038/ng.3737

44. Raj, T. et al. Polarization of the Effects of Autoimmune and Neurodegenerative Risk Alleles in Leukocytes. Science (80-. ). 344, 519523 (2014).

45. Ernst, J. \& Kellis, M. ChromHMM: automating chromatin-state discovery and characterization. Nat. Methods 9, 215-216 (2012).

46. Roadmap Epigenomics Consortium, A. et al. Integrative analysis of 111 reference human epigenomes. Nature 518, 317-30 (2015).

47. Ward, L. D. \& Kellis, M. HaploReg v4: systematic mining of putative causal variants, cell types, regulators and target genes for human complex traits and disease. Nucleic Acids Res. 44, D877-D881 (2016).

48. Buenrostro, J. D., Giresi, P. G., Zaba, L. C., Chang, H. Y. \& Greenleaf, W. J. Transposition of native chromatin for fast and sensitive epigenomic profiling of open chromatin, DNA-binding proteins and nucleosome position. Nat. Methods 10, 1213-8 (2013).

49. Zhang, Y. et al. Model-based analysis of ChIP-Seq (MACS). Genome Biol. 9, R137 (2008). 
Figure 1

A

Coverage above

B

Total coverage threshold

Coverage increase

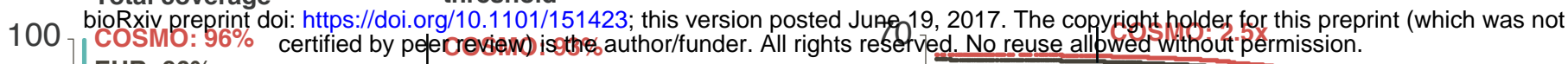
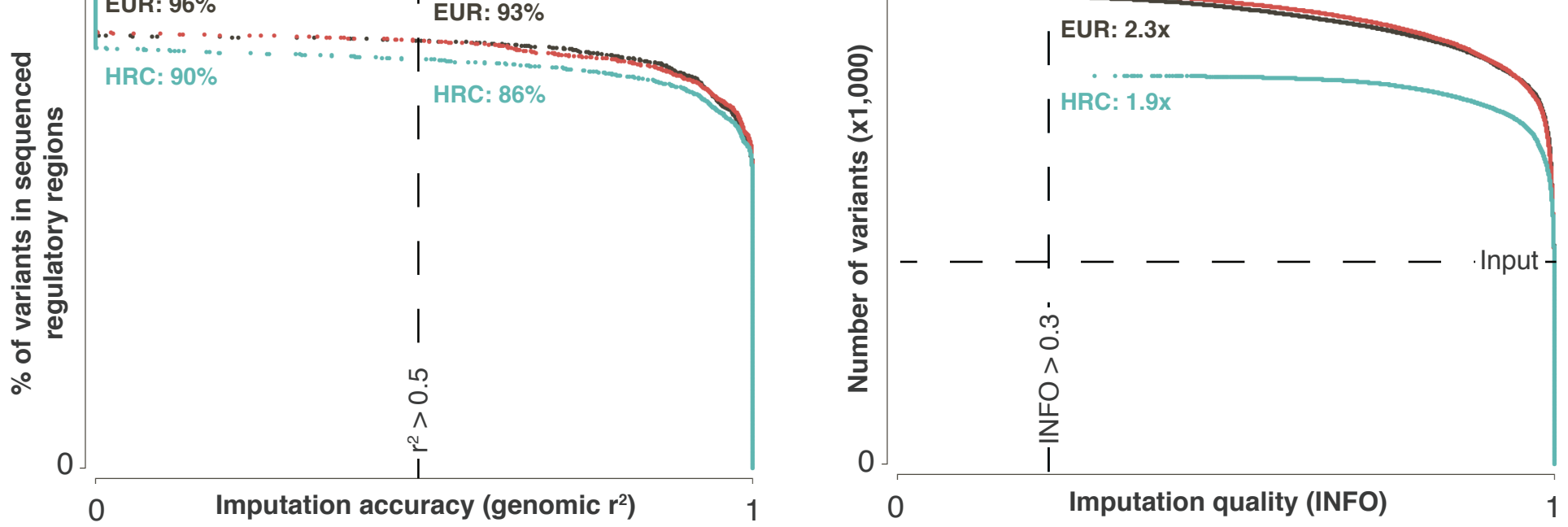


\section{Figure 2}

A
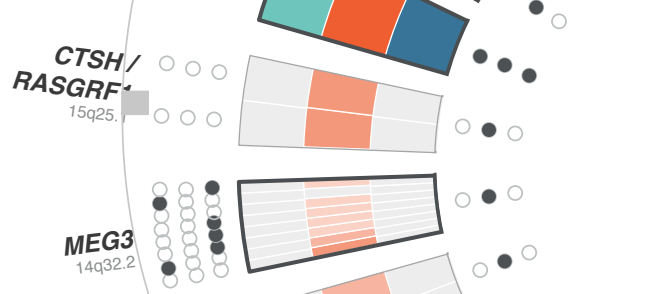
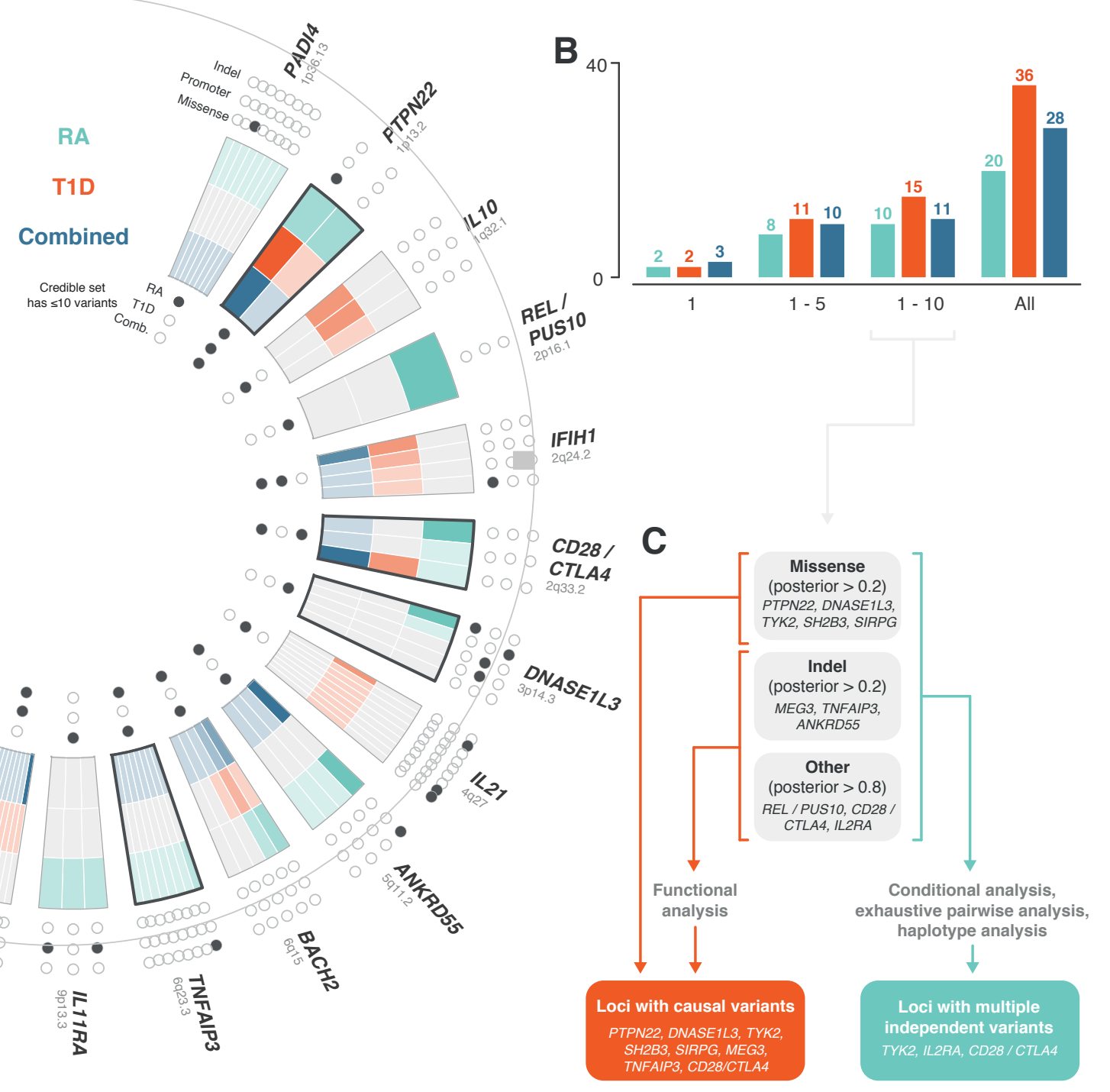

RA

Not associated \begin{tabular}{|l|l|l|}
\hline$\square$ & T1D \\
\hline
\end{tabular}

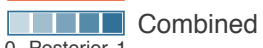


bioRxiv preprint doi: https://doi.org/10.1101/151423; this version posted June 19, 2017. The copyright holder for this preprint (which was not

Figure 3 - CD28\%"CTL'A4"

A RA, T1D Combined
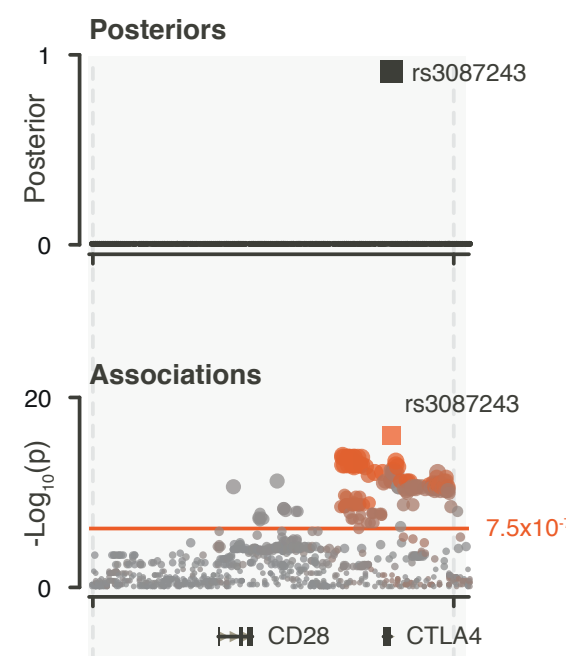

$204.45 \quad$ Chr $2(\mathrm{mb})$
$\begin{gathered}\text { Linkage } \\ \text { Disequilibrium }\end{gathered}$

C

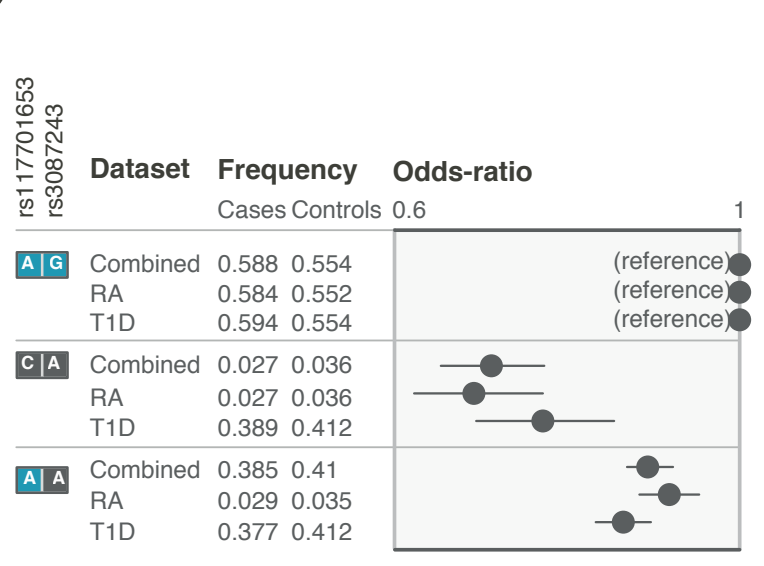

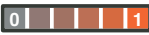

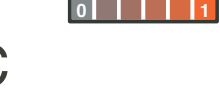

\section{B Rheumatoid Arthritis}
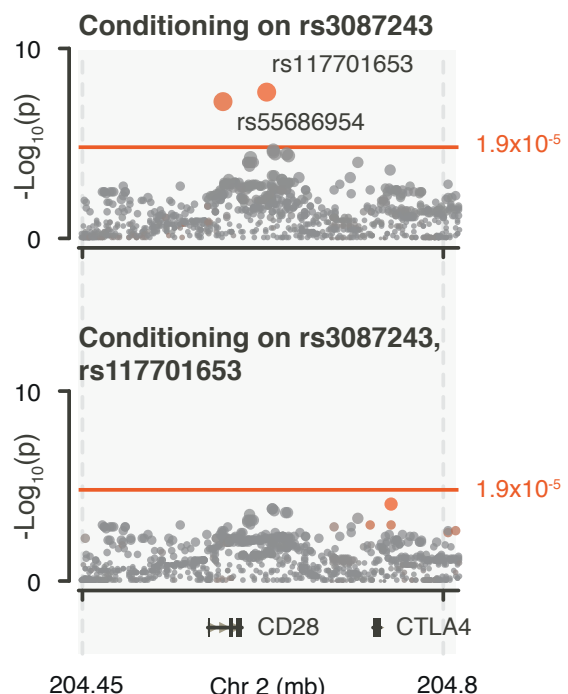

Chr $2(\mathrm{mb})$

204.8

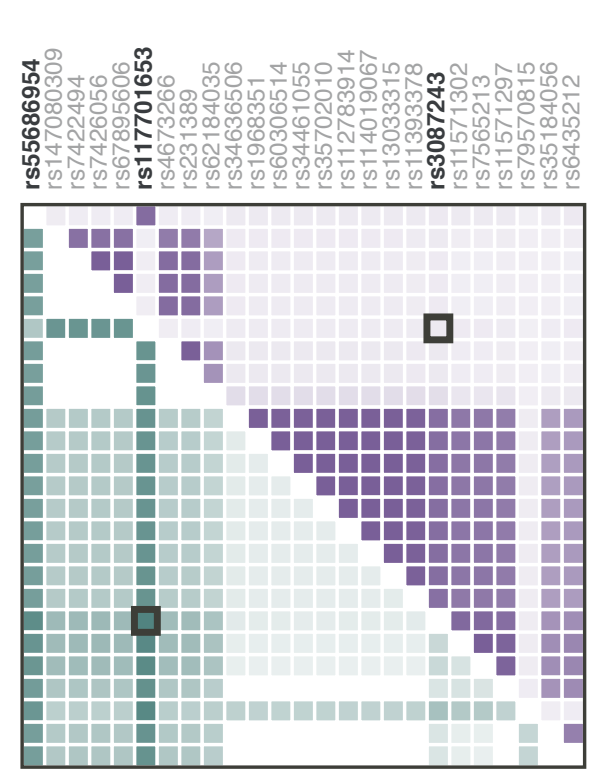

rs3087243 + rs117701653: $\log 10(p)=13.81$ Most significant pair out of 567,629 -log10(p-value)

\begin{tabular}{|l|l|}
\hline & 14 \\
\hline 6
\end{tabular}

Linkage Disequilibrium \begin{tabular}{|l|l|}
\hline$\square$ \\
\hline
\end{tabular}

rs55686954 rs147080309 rs74722494
rs7426056 rs7426056 rs117701653 is4673266 rs 231389 rs62184035 rs34636506 rs1968351 rs60306514 rs34461055 rs35702010 rs112783914 rs114019067 rs13033315 rs3087243 rs11571302 rs7565213 rs11571297 rs79570815 rs35184056 rs6435212
D rs117701653 rs3087243 (Jurkat) (Jurkat)

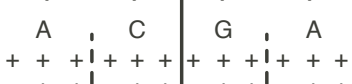

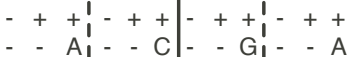

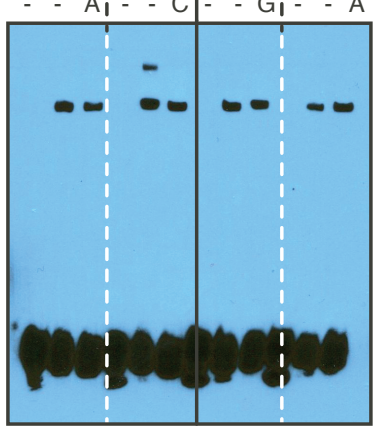

$E$

Probe allele

Biotin probe

Protein sample

Non-biotin probe

$\longleftarrow$ Specific

$\leftarrow$ Non-specific binding

$\leftarrow$ Free biotin probe

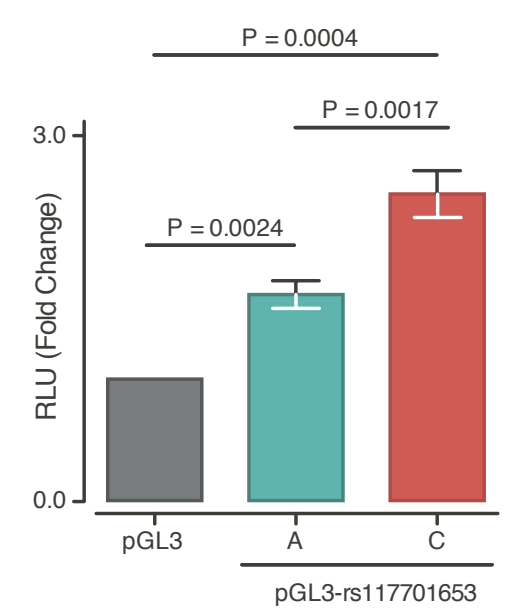


bioRxiv preprint doi: https://doi.org/10.1101/151423; this version posted June 19, 2017. The copyright holder for this preprint (which was not

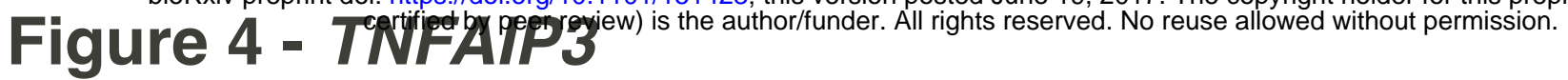

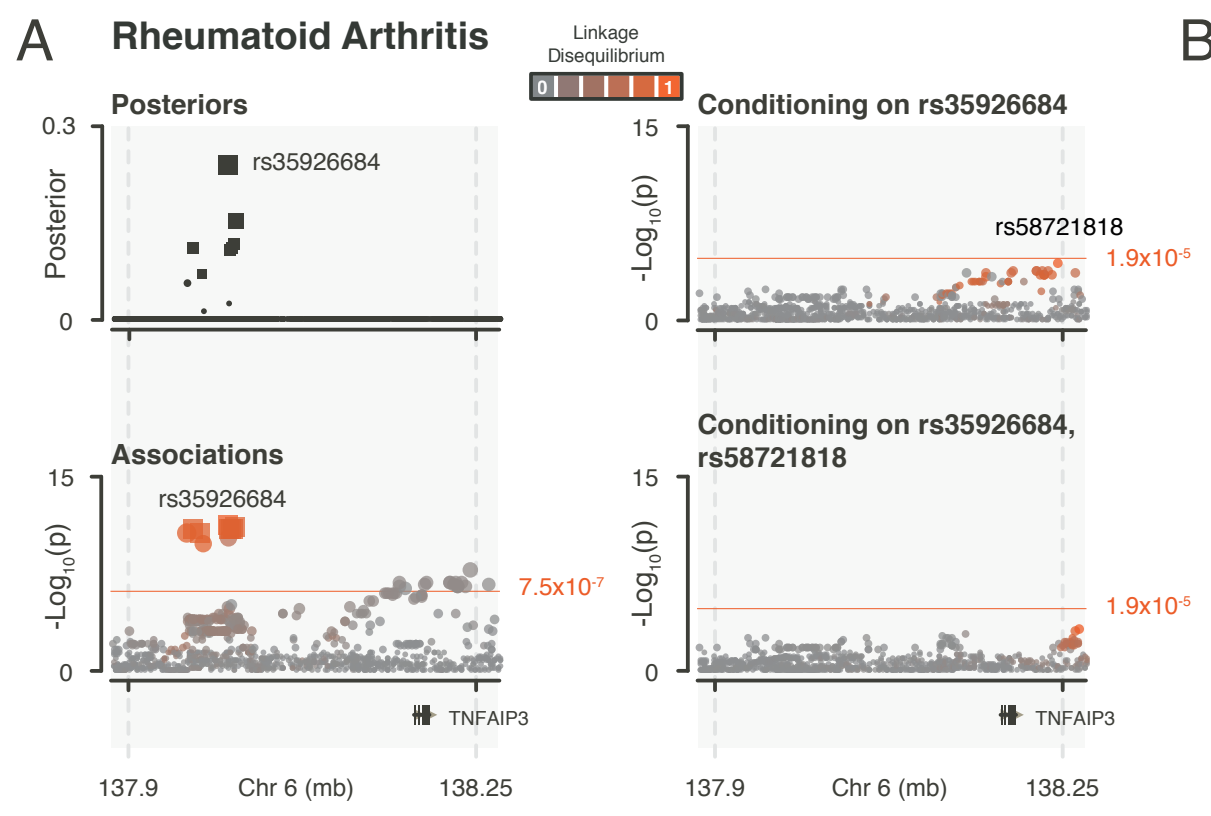

C

\begin{tabular}{|c|c|c|}
\hline & Frequ & ency \\
\hline 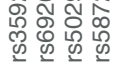 & Cases & Controls \\
\hline 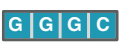 & 0.743 & 0.771 \\
\hline \begin{tabular}{l|l|l|l|}
$G|G|$ &
\end{tabular} & 0.014 & 0.011 \\
\hline \begin{tabular}{l|l|l|l|}
$G|A| G \mid$ &
\end{tabular} & 0.217 & 0.198 \\
\hline \begin{tabular}{|l|}
$\mathbf{G}|\mathbf{A}| \mathbf{T} \mid$ \\
\end{tabular} & 0.026 & 0.02 \\
\hline
\end{tabular}

Odds-ratio

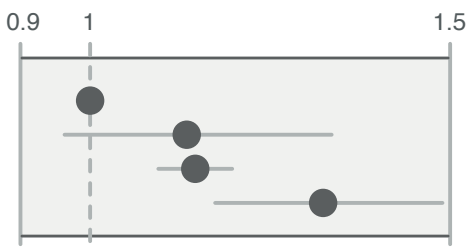

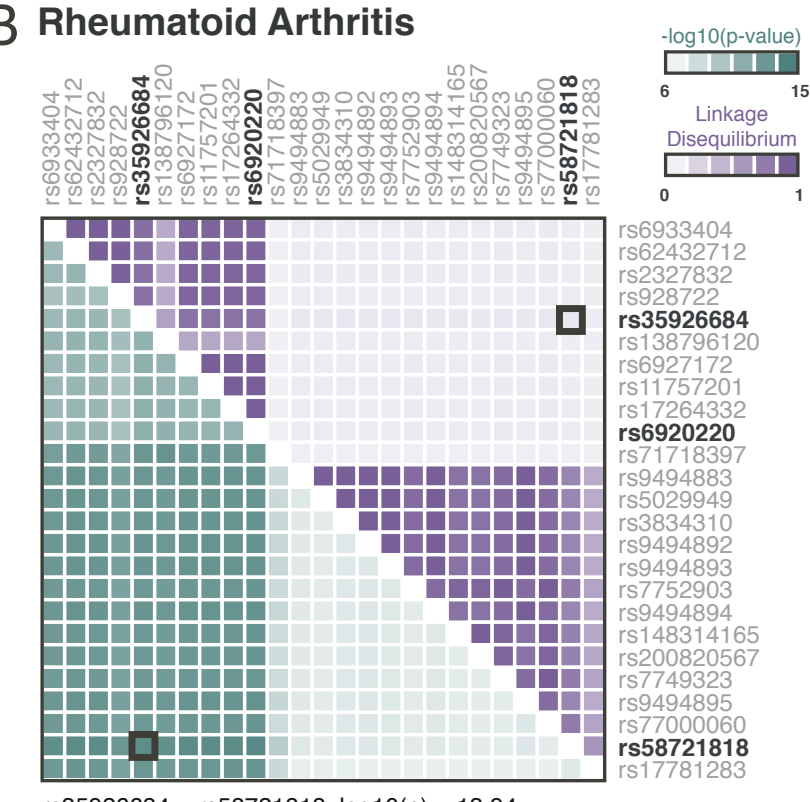

rs35926684 + rs58721818, $\log 10(p)=13.94$

rs35926684 + rs58721818 is the seventh most significant pair of 697,970 pairs; Top pair: $r s 6920220+r s 58721818, \log 10(p)=14.21$
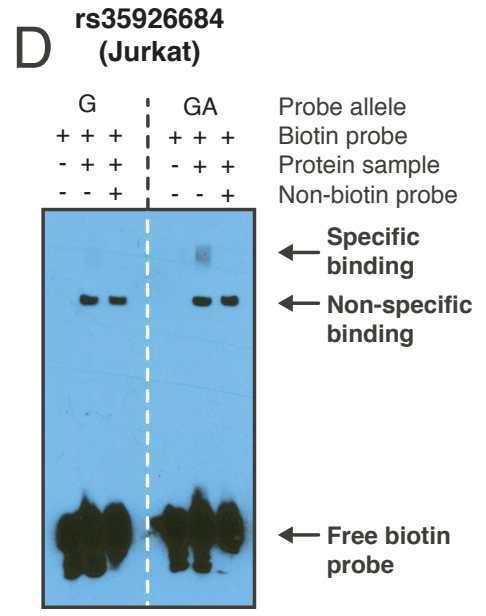

$E$

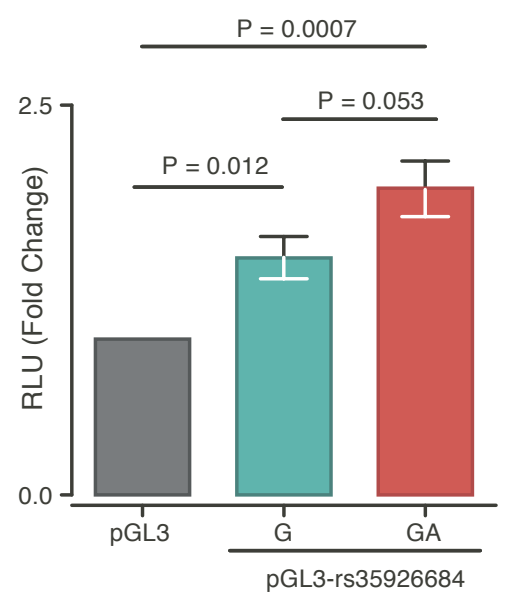


bioRxiv preprint doi: https://doi.org/10.1101/151423; this version posted June 19, 2017. The copyright holder for this preprint (which was not

Figure 5 - MEEG

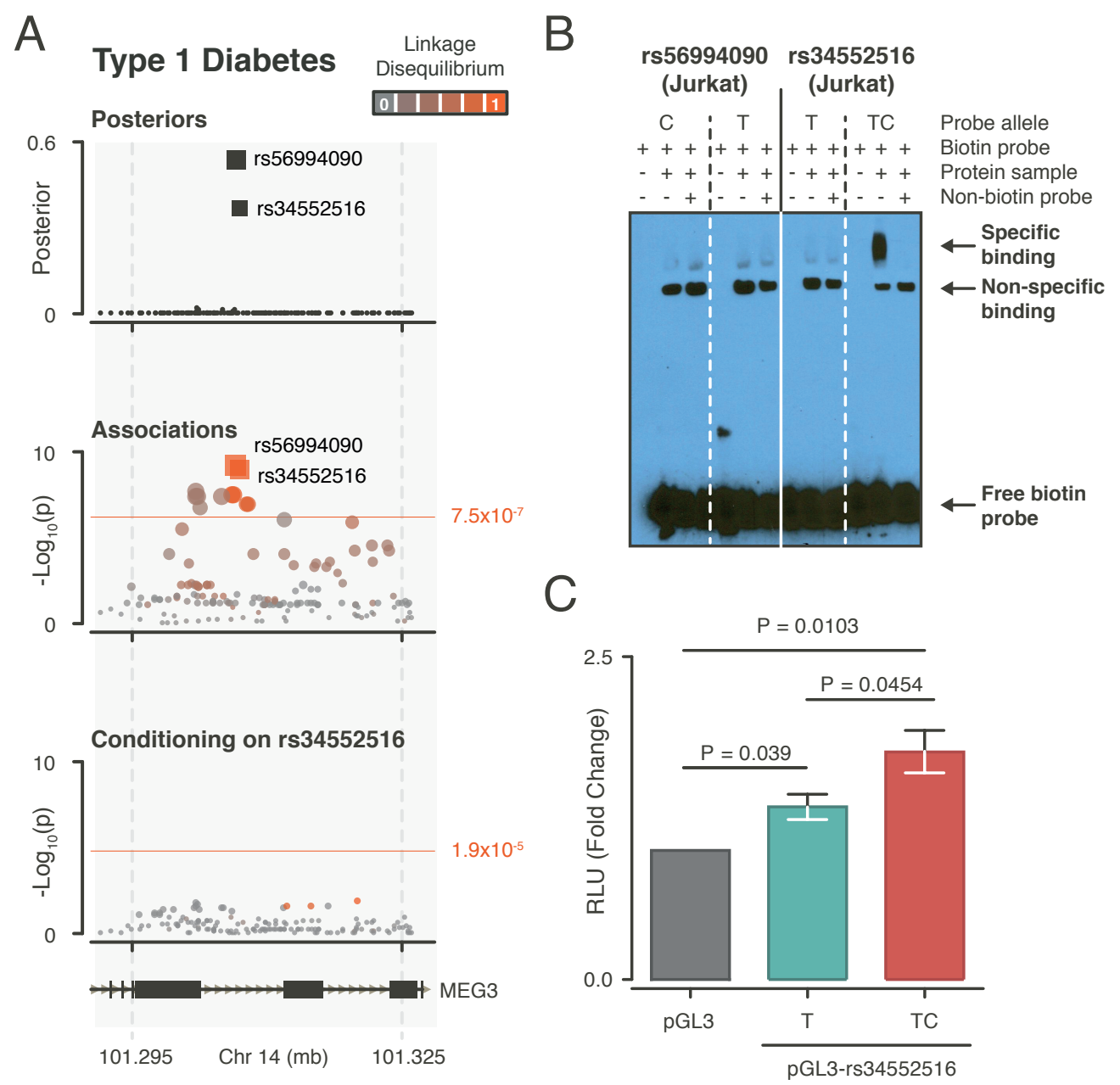

